\section{Labour market}

\section{Labour Force Survey}

(Tables 7.1 to $7.3,7.6,7.9,7.10$ to $7.11,7.13$ and 7.16 to 7.18$)$

\section{The impact of Census 2001 on LFS data}

(Tables 7.1 to $7.3,7.6,7.9,7.10$ to $7.11,7.16$ to 7.18 )

The first results of the 2001 Census, published on 30 September 2002, showed that previous estimates of the total UK population were about one million too high. Estimates of employment and unemployment levels from the Labour Force Survey (LFS) released before 30 October 2002 were therefore too high, with rates also affected. This led to the LFS needing to reweight its estimates to the new population figures.

Top-level seasonally adjusted interim reweighed figures were published in the 2003 edition of Annual Abstract of Statistics. LFS estimates for the UK, used in this chapter, continue to show seasonally adjusted data for these tables, in line with other headline publications. These figures are not directly comparable to the non -seasonally adjusted figures published in the Annual Abstract of Statistics before 2003.

Since 2003, the LFS aggregate estimates (that is, the key labour market indicators, for example, the levels and rates of employment, unemployment and economic inactivity) have been interim reweighted every year. Interim reweighting applies adjustments to the aggregate results to reflect how the latest available LFS household population estimates compare with those used for weighting the microdata. This amounts to an approximation of the effect that a full reweighting of the microdata would have.

A full reweighting exercise was carried out in 2007-08 and the results were published on 14 May 2008. The microdatasets were weighted to the latest (2007/08) population estimates whereas previously they were based on population totals published in 2003. For the previous reweighting exercise the microdata were weighted to Census 2001 population estimates published in spring 2003. In autumn 2003 these estimates were revised but it was too late to incorporate them into the reweighting without significantly delaying the project. Therefore, it was decided that the weights already produced using the spring 2003 estimates would be retained and that projections from the pre-revised Census would then be used to weight subsequent datasets until 2007.

Up-to-date population estimates that are in line with the revised Census figures have been used in the latest reweighting project. Analysis of the 2007 reweighted microdata produced aggregates at the UK level that are approximately 900,000 above the estimates produced from the datasets weighted to the 2003 population totals. The changes in the estimates are primarily due to overall differences between the 2003 and 2007 population totals used to weight the data. However, the underlying weighting methodology was changed for the 2007 reweighting exercise, which is another factor that has contributed to changes in the reweighted estimates.

For more information on the reweighting exercise, see 'Labour Force Survey: reweighting and seasonal adjustment review 2008' available at: www.statistics.gov.uk/CCl/article. asp?|D=2011 and 'Labour Force Survey: Interim reweighting 2008 available at: www.statistics.gov.uk/cci/article. asp? $\mathrm{id}=2061$

\section{Background}

The LFS is the largest regular household survey in the UK LFS interviews are conducted continuously throughout the year. In any three-month period, a nationally representative sample of approximately 102,000 people aged 16 or over in around 52,000 households are interviewed. Each household is interviewed five times, at three-monthly intervals. The initial interview is done face-to-face by an interviewer visiting the address. The other interviews are done by telephone wherever possible. The survey asks a series of questions about respondents' personal circumstances and their labour market activity. Most questions refer to activity in the week before the interview.

The concepts and definitions used in the LFS are agreed by the International Labour Organisation (ILO) - an agency of the United Nations. The definitions are used by European Union member countries and members of the Organisation for Economic Co-operation and Development (OECD).

\section{Labour Market}

The LFS was carried out every two years from 1973 to 1983. The ILO definition was first used in 1984. This was also the first year in which the survey was conducted on an annual basis with results available for every spring quarter (representing an average of the period from March to May). The survey moved to a continuous basis in spring 1992 in Great Britain and in winter 1994/95 in Northern Ireland, with average quarterly results published four times a year for seasonal quarters: spring (March to May), summer (June to August), autumn (September to November) and winter (December to February). From April 1998, results are published 12 times a year for the average of three consecutive months.

The LFS collects information on a sample of the population. To convert this information to give estimates for the population the data must be grossed. This is achieved by calculating weighting factors (often referred to simply as weights) which 
can be applied to each sampled individual in such a way that the weighted-up results match estimates or projections of the total population in terms of age distribution, sex, and region of residence.

\section{Strengths and limitations of the LFS}

The LFS produces coherent labour market information on the basis of internationally standard concepts and definitions. It is a rich source of data on a wide variety of labour market and personal characteristics. It is the most suitable source for making comparisons between countries. The LFS is designed so that households interviewed in each three month period constitute a representative sample of UK households. The survey covers those living in private households and nurses in National Health Service accommodation. Students living in halls of residence have been included since 1992 as information about them is collected at their parents' address.

However the LFS has its limitations. It is a sample survey and is therefore subject to sampling variability. The survey does not include people living in institutions such as hostels or residential homes. 'Proxy' reporting (when members of the household are not present at the interview, another member of the household answers the questions on their behalf) can affect the quality of information on topics such as earnings, hours worked, benefit receipt and qualifications. Around one-third of interviews are conducted 'by proxy', usually by a spouse or partner but sometimes by a parent or other near relation.

\section{Sampling variability}

Survey estimates are prone to sampling variability. The easiest way to explain this concept is by example. In the September to November 1997 period, International Labour Organisation (ILO) unemployment in Great Britain (seasonally adjusted) stood at $1,847,000$. If we drew another sample for the same period we could get a different result, perhaps $1,900,000$ or $1,820,000$.

In theory, we could draw many samples, and each would give a different result. This is because each sample would be made up of different people who would give different answers to the questions. The spread of these results is the sampling variability. Sampling variability is determined by a number of factors including the sample size, the variability of the population from which the sample is drawn and the sample design. Once we know the sampling variability we can calculate a range of values about the sample estimate that represents the expected variation with a given level of assurance. This is called a confidence interval. For a 95 per cent confidence interval we expect that in 95 per cent of the samples (19 times out of 20 ) the confidence interval will contain the true value that would be obtained by surveying the entire population. For the example given above, we can be 95 per cent confident that the true value was in the range $1,791,000$ to $1,903,000$.

\section{Unreliable estimates}

Very small estimates have relatively wide confidence intervals making them unreliable. For this reason, ONS does not publish LFS estimates below 10,000 .

\section{Non-response}

Non-response can introduce bias to a survey, particularly if the people not responding have characteristics that are different from those who do respond. The LFS has a response rate of around 80 per cent to the first interview, and over 90 per cent of those who are interviewed once go on to complete all five interviews. These are relatively high levels for a household survey. Any bias from non-response is minimised by weighting the results.

Weighting (or grossing) converts sample data to represent the full population. In the LFS, the data are weighted separately by age, sex and area of residence to population estimates based on the census. Weighting also adjusts for people not in the survey and thus minimises non-response bias.

\section{LFS concepts and definitions}

Discouraged workers - a sub-group of the economically inactive population, defined as those neither in employment nor unemployed (on the ILO measure) who said they would like a job and whose main reason for not seeking work was because they believed there were no jobs available.

Economically active - people aged 16 and over who are either in employment or ILO unemployed.

Economic activity rate - the percentage of people aged 16 and over who are economically active.

Economically inactive - people who are neither in employment nor unemployed. This group includes, for example, all those who were looking after a home or retired.

Employment - people aged 16 or over who did at least one hour of paid work in the reference week (whether as an employee or self-employed); those who had a job that they were temporarily away from (on holiday, for example); those on government-supported training and employment programmes (from spring 1983); and those doing unpaid family work (from spring 1992). 
Employees - the division between employees and self-employed is based on survey respondents' own assessment of their employment status.

Full Time - the classification of employees, self-employed and unpaid family workers in their main job as full-time or part-time is on the basis of self-assessment. Up until autumn 1995, people who were on government workrelated training programmes are classified as full-time or part-time according to whether their usual hours of work per week were over 30 or 30 and under; from winter 1995/96 onwards, the full-time/part-time classification for this group has been changed to self-assessment, in line with the other groups outlined above. People on government-supported training and employment programmes who are at college in the survey reference week are classified, by convention, as part-time.

Government-supported training and employment programmes - comprise all people aged 16 and over participating in one of the government's employment and training programmes (Youth Training, Training for Work and Community Action), together with those on similar programmes administered by Training and Enterprise Councils in England and Wales, or Local Enterprise Companies in Scotland.

Hours worked - respondents to the LFS are asked a series of questions enabling the identification of both their usual hours and their actual hours. Total hours include overtime (paid and unpaid) and exclude lunch-breaks.

Unemployment - Unemployment figures from the LFS, which are based upon the ILO definition, were re-labelled 'unemployment' rather than 'ILO unemployment' in January 2003. This emphasises that the LFS figures provide the official and only internationally comparable measure of unemployment in the UK. For more details see: www. statistics.gov.uk/cci/nugget.asp? $\mathrm{id}=251$

The ILO measure of unemployment used throughout this publication refers to people without a job who were available to start work in the two weeks following their LFS interview and who had either looked for work in the four weeks prior to interview or were waiting to start a job they had already obtained. This definition of unemployment is in accordance with that adopted by the 13th International Conference of Labour Statisticians, further clarified at the 14th ICLS, and promulgated by the ILO in its publications.

Unemployment (rate) - the percentage of economically active people who are unemployed on the ILO measure.

Unemployment (duration) - defined as the shorter of the following two periods:
- duration of active search for work

- $\quad$ length of time since employment

Part-time - see full-time.

Second jobs - jobs which LFS respondents hold in addition to a main full-time or part-time job.

Self-employment - See Employees.

Temporary employees - in the LFS these are defined as those employees who say that their main job is non-permanent in one of the following ways: fixed period contract; agency temping; casual work; seasonal work; other temporary work.

Unpaid family workers - the separate identification from spring 1992 of this group in the LFS is in accordance with international recommendations. The group comprises persons doing unpaid work for a business they own or for a business that a relative owns.

\section{Distribution of workforce}

\section{(Table 7.4)}

Claimant unemployed - those people who were claiming unemployment-related benefits (contributions or income related Jobseeker's Allowance and/or National Insurance credits) at Jobcentre Plus local offices on the day of the monthly count. The seasonally adjusted claimant unemployment series allows for all relevant changes which, unless adjusted for, would distort comparisons over time.

Workforce jobs (formerly workforce in employment) - comprises employee jobs, self-employment jobs (from the LFS), HM Forces and government-supported trainees.

HM Forces (provided by Ministry of Defence) - represent the total number of UK service personnel, male and female, in HM Regular. Full Time Reserve personnel, mobilised reservists, the Ghurkhas and the Home Service battalions of the Royal Irish Regiment, wherever serving and including those on leave.

Self-employed jobs - estimates are based on the results of the Labour Force Survey. The Northern Ireland estimates are not seasonally adjusted.

Government-supported trainees - include all participants on government training and employment programmes who are receiving some work experience on their placement but who do not have a contract of employment (those with a contract are included in the employee jobs series). The numbers are not subject to seasonal adjustment. 


\section{International Employment Comparisons}

\section{(Table 7.7)}

All employment rates for European Union (EU) countries published by Eurostat (including the rate for the UK) are based on the population aged 15-64. The rates for Canada and Japan are also based on the population aged 15-64, but the rate for the US is for those aged 16-64. The employment rate for the UK published by ONS is based on the working age population aged 16-64 (men) and 16-59 (women) and therefore takes into account both the current school leaving age and state pension ages.

The unemployment rate published by Eurostat for most EU countries (but not for the UK), are calculated by extrapolating from the most recent LFS data using monthly registered unemployment data. A standard population basis (15-74) is used by Eurostat except for Spain and the UK (16-74). The unemployment rate for the US is based on those aged 16 and over, but the rates for Canada and Japan are for those aged 15 and over. All unemployment rates are seasonally adjusted.

The unemployment rate for the UK published by Eurostat is based on the population aged 16-74 while the unemployment rate for the UK published by ONS is based on those aged 16 and over. There are other minor definitional differences.

\section{Jobseekers allowance claimant count}

\section{(Tables 7.14 and 7.15)}

This is a count of all those people who are claiming Jobseeker's Allowance (JSA) at Jobcentre Plus local offices. People claiming JSA must declare that they are:

- out of work

- capable of work

- $\quad$ available for work

- $\quad$ actively seeking work

during the week in which the claim is made.

All people claiming JSA on the day of the monthly count are included in the claimant count, irrespective of whether they are actually receiving benefits. Also see Table 10.6 in the Social protection chapter.

\section{Labour disputes}

(Table 7.19)

These figures exclude details of stoppages involving fewer than ten workers or lasting less than one day except any in which the aggregate number of working days lost is 100 or more. There may be some under-recording of small or short stoppages; this would have much more effect on the total of stoppages than of working days lost. Some stoppages which affected more than one industry group have been counted under each of the industries but only once in the totals. Stoppages have been classified using the Standard Industrial Classification (SIC) 2003.

The figures for working days lost and workers involved have been rounded and consequently the sum of the constituent items may not agree with the totals. Classifications by size are based on the full duration of stoppages where these continue into the following year. Working days lost per thousand employees are based on the latest available mid-year (June) estimates of employee jobs.

\section{Earnings}

\section{(Tables 7.20 to 7.25 )}

The total gross remuneration employees receive before any statutory deductions (tax, national insurance). Income in kind and pension funds are excluded.

\section{Annual Survey of Hours and Earnings}

(Tables 7.20, 7.21, 7.24 and 7.25)

The Annual Survey of Hours and Earnings (ASHE) replaced the New Earnings Survey (NES) in 2004. The ASHE includes improvements to the coverage of employees and to the weighting of earnings estimates. The data variables collected remain broadly the same, although an improved questionnaire was introduced for the 2005 survey. The change in methodology means that statistics on pay and hours published from the ASHE, including the calculation of ONS's low pay statistics, are discontinuous with previous NES surveys.

To improve coverage and make the survey more representative, supplementary information was collected for the 2004 ASHE survey on businesses not registered for Value Added Tax (VAT) and for people who changed or started new jobs between sample selection and the survey reference period. The 2004 ASHE results are therefore discontinuous with the results for 2003, for which no supplementary information was collected. However, for 2004 two sets of results are available; the headline results that include supplementary information and results that exclude this 
information. These second set of results are given solely for comparison to earlier results.

The ASHE methodology includes imputation and weighting, the main impact of these changes when applied to existing NES data for 1997 to 2003 are:

- to increase the estimates of the level of average weekly pay over estimates published from the NES

- for males the increase in estimates of earnings is more than the increase for females. In particular this affects hourly pay excluding overtime, which is used in the calculation of ONS's preferred measure of the gender pay gap. The estimate of hourly pay for males is increased more then the estimate for females, which widens the estimate of the gap between male and female hourly pay

- $\quad$ estimates of the level of earnings for people working in London are increased more than estimates for other regions. This widens the estimate of the difference in pay between London and other regions of the UK

For information about methodological changes to the 2007 ASHE survey see: www.statistics.gov.uk/downloads/theme_ labour/ASHE/ChangelnASHE07.pdf

\section{Average Earnings Index}

\section{(Tables 7.22 and 7.23)}

The Average Earnings Index (AEI) is designed to measure changes in the level of earnings, that is, wage inflation in Great Britain. Average earnings are calculated as the total wages and salaries paid by firms, divided by the number of employees paid. Like all indices, changes are measured against a base year, whose index value is set to 100 . The current base year is 2000 for Tables 7.22 and 7.23.

Users should note that the data contained in Table 7.23 of the Annual Abstract of Statistics since 2003 are not comparable with that published up until 2002. Table 7.23 now shows the set of 20 industry sectors that better reflect the current state of the economy, and supersedes the previous set of 26 industry sectors. The new series are available in the format of excluding bonus index, including bonus index, and an annual percentage change for including and excluding bonuses. An article covering the reasons for the change can be found at: www.statistics.gov.uk/labour

The AEl is published monthly in the ONS Labour Market Statistics First Release. The main indicator of growth, the headline rate, is based on the annual change in the seasonally adjusted index values for the latest three months compared with the same period a year ago. The use of a three-month average reduces the level of volatility seen in the data on a month-onmonth basis.

\section{Strengths of the AEI}

The AEl, based on monthly survey data, is a timely indicator of changes in the level of earnings.

\section{Limitations of the AEI}

The index is not adjusted for any changes in the composition of the workforce such as changes in the share of full-time and part-time workers, or in the share of skilled and unskilled workers. Similarly, the index does

not account for changes in the number of hours worked, or any temporary factors that affect earnings.

The sample of the Monthly Wages and Salaries Survey on which the AEl is based is not designed to provide information on the level of earnings. The sample is not completely representative of the economy as firms with fewer than 20 employees are excluded, as are the earnings of self-employed persons.

The AEl only covers earnings in Great Britain as earnings information is not collected for Northern Ireland and regional data are not available.

\section{Trade unions}

\section{(Table 7.26)}

The statistics relate to all organisations of employees known to Certification Officer with head offices in the UK that fall within the appropriate definition of a trade union in the Trade Union and Labour Relations (Consolidation) Act 1992. Included in the data are home and overseas membership figures of contributory and non-contributory members. Employment status of members is not provided and the figures may therefore include some people who are self-employed, unemployed or retired.

The membership part of this table was revised in 2001, so that statistics presented here are on a consistent basis with the Great Britain table produced by the Certification Officer in his Annual Report and with tables produced in the annual Trade Union membership article published by the Department for Business, Enterprise \& Regulatory Reform (BERR). 
Labour Market

\begin{tabular}{|c|c|c|c|c|c|c|c|c|c|}
\hline & \multicolumn{5}{|c|}{ All aged 16 and over } & \multicolumn{4}{|c|}{ Percentages } \\
\hline & Total $^{3}$ & $\begin{array}{r}\text { Total } \\
\text { economically } \\
\text { active }\end{array}$ & $\begin{array}{r}\text { Total in } \\
\text { employment }\end{array}$ & $\begin{array}{r}\text { Total } \\
\text { unemployed }\end{array}$ & $\begin{array}{r}\text { Economically } \\
\text { inactive }\end{array}$ & $\begin{array}{c}\text { Economic } \\
\text { activity rate } \\
16-59 / 64^{4}\end{array}$ & $\begin{array}{r}\text { Employment rate } \\
\text { all aged } 16 \text { and } \\
\text { over }^{5}\end{array}$ & $\begin{array}{r}\text { Employment rate } \\
16-59 / 64^{6}\end{array}$ & $\begin{array}{r}\text { Unemployment } \\
\text { rate }^{7}\end{array}$ \\
\hline \multicolumn{10}{|c|}{$\begin{array}{l}\text { All } \\
\text { Persons }\end{array}$} \\
\hline & MGSL & MGSF & MGRZ & MGSC & MGSI & MGSO & MGSR & MGSU & MGSX \\
\hline 1998 & 45720 & 28583 & 26795 & 1791 & 17137 & 78.4 & 58.6 & 73.4 & 6.3 \\
\hline 1999 & 45937 & 28840 & 27167 & 1743 & 17065 & 78.7 & 59.2 & 74.0 & 6.0 \\
\hline 2000 & 46194 & 29069 & 27483 & 1600 & 17084 & 78.8 & 59.5 & 74.4 & 5.5 \\
\hline 2001 & 46502 & 29176 & 27710 & 1470 & 17291 & 78.6 & 59.6 & 74.4 & 5.0 \\
\hline 2002 & 46787 & 29433 & 27921 & 1521 & 17317 & 78.6 & 59.7 & 74.5 & 5.2 \\
\hline 2003 & 47087 & 29659 & 28186 & 1468 & 17382 & 78.7 & 59.9 & 74.6 & 5.0 \\
\hline 2004 & 47448 & 29867 & 28485 & 1439 & 17524 & 78.5 & 60.0 & 74.7 & 4.8 \\
\hline 2005 & 47871 & 30170 & 28774 & 1438 & 17653 & 78.5 & 60.1 & 74.7 & 4.8 \\
\hline 2006 & 48268 & 30686 & 29030 & 1687 & 17531 & 79.0 & 60.2 & 74.6 & 5.5 \\
\hline 2007 & 48668 & 30821 & 29222 & 1662 & 17800 & 78.8 & 60.0 & 74.6 & 5.4 \\
\hline 2008 & 49059 & 31190 & 29443 & 1685 & 17816 & 79.1 & 60.0 & 74.5 & 5.4 \\
\hline \multicolumn{10}{|l|}{ Male } \\
\hline & MGSM & MGSG & MGSA & MGSD & MGSJ & MGSP & MGSS & MGSV & MGSY \\
\hline 1998 & 21992 & 15685 & 14597 & 1076 & 6308 & 84.3 & 66.4 & 78.4 & 6.9 \\
\hline 1999 & 22110 & 15795 & 14767 & 1060 & 6298 & 84.4 & 66.8 & 78.8 & 6.7 \\
\hline 2000 & 22249 & 15860 & 14909 & 959 & 6365 & 84.4 & 67.0 & 79.2 & 6.0 \\
\hline 2001 & 22430 & 15883 & 15026 & 882 & 6525 & 83.9 & 67.0 & 79.3 & 5.6 \\
\hline 2002 & 22600 & 15988 & 15099 & 914 & 6591 & 83.8 & 66.8 & 79.0 & 5.7 \\
\hline 2003 & 22775 & 16187 & 15262 & 890 & 6562 & 84.1 & 67.0 & 79.2 & 5.5 \\
\hline 2004 & 22978 & 16217 & 15405 & 845 & 6731 & 83.6 & 67.1 & 79.3 & 5.2 \\
\hline 2005 & 23214 & 16352 & 15535 & 837 & 6835 & 83.4 & 66.9 & 79.0 & 5.1 \\
\hline 2006 & 23438 & 16606 & 15662 & 978 & 6804 & 83.7 & 66.9 & 78.8 & 5.9 \\
\hline 2007 & 23668 & 16742 & 15813 & 950 & 6899 & 83.6 & 66.8 & 78.8 & 5.7 \\
\hline 2008 & 23891 & 16928 & 15894 & 990 & 6934 & 83.7 & 66.5 & 78.5 & 5.8 \\
\hline \multicolumn{10}{|c|}{ Female } \\
\hline & MGSN & MGSH & MGSB & MGSE & MGSK & MGSQ & MGST & MGSW & MGSZ \\
\hline 2000 & 23945 & 13209 & 12573 & 641 & 10719 & 72.9 & 52.5 & 69.3 & 4.9 \\
\hline 2001 & 24072 & 13292 & 12684 & 589 & 10766 & 72.9 & 52.7 & 69.3 & 4.4 \\
\hline 2002 & 24186 & 13445 & 12823 & 607 & 10726 & 73.1 & 53.0 & 69.6 & 4.5 \\
\hline 2003 & 24311 & 13472 & 12925 & 579 & 10819 & 72.9 & 53.1 & 69.7 & 4.3 \\
\hline 2004 & 24469 & 13650 & 13080 & 593 & 10793 & 73.1 & 53.5 & 69.9 & 4.3 \\
\hline 2005 & 24657 & 13819 & 13239 & 601 & 10818 & 73.3 & 53.7 & 70.0 & 4.4 \\
\hline 2006 & 24830 & 14080 & 13367 & 709 & 10728 & 74.0 & 53.8 & 70.0 & 5.0 \\
\hline 2007 & 25001 & 14079 & 13409 & 712 & 10901 & 73.6 & 53.7 & 69.9 & 5.1 \\
\hline 2008 & 25168 & 14262 & 13549 & 695 & 10882 & 74.1 & 53.8 & 70.2 & 4.9 \\
\hline
\end{tabular}

1 See chapter text. In August 2007, ONS published the mid-year population estimates for 2006. These estimates have now been incorporated into the LFS estimates from 2001 onwards. Further details can be found at http://www.statistics.gov.uk/cci/article. asp?id=1919

2 The Labour Force Survey has now moved to calendar quarters from May 2006. More information can be found on page 5 of the Concepts and Definitions.pdf by following this link:- www.statistics.gov.uk/downloads/theme_labour/Concepts_Definitions_HQS.pdf

3 Population aged 16 and over in private households and student halls of residence.

4 Economically active of working age as a percentage of all persons of working age (men 16-64, women 16-59).

5 Total employed as a percentage of all persons aged 16 and over.

6 Total employed of working age as a percentage of all persons of working age (men 16-64, women 16-59).

7 Total unemployed as a percentage of all economically active. 
72 Employment status: full-time, part-time and temporary employees ${ }^{1}$

United Kingdom

At Quarter 2 each year ${ }^{2}$. Seasonally adjusted

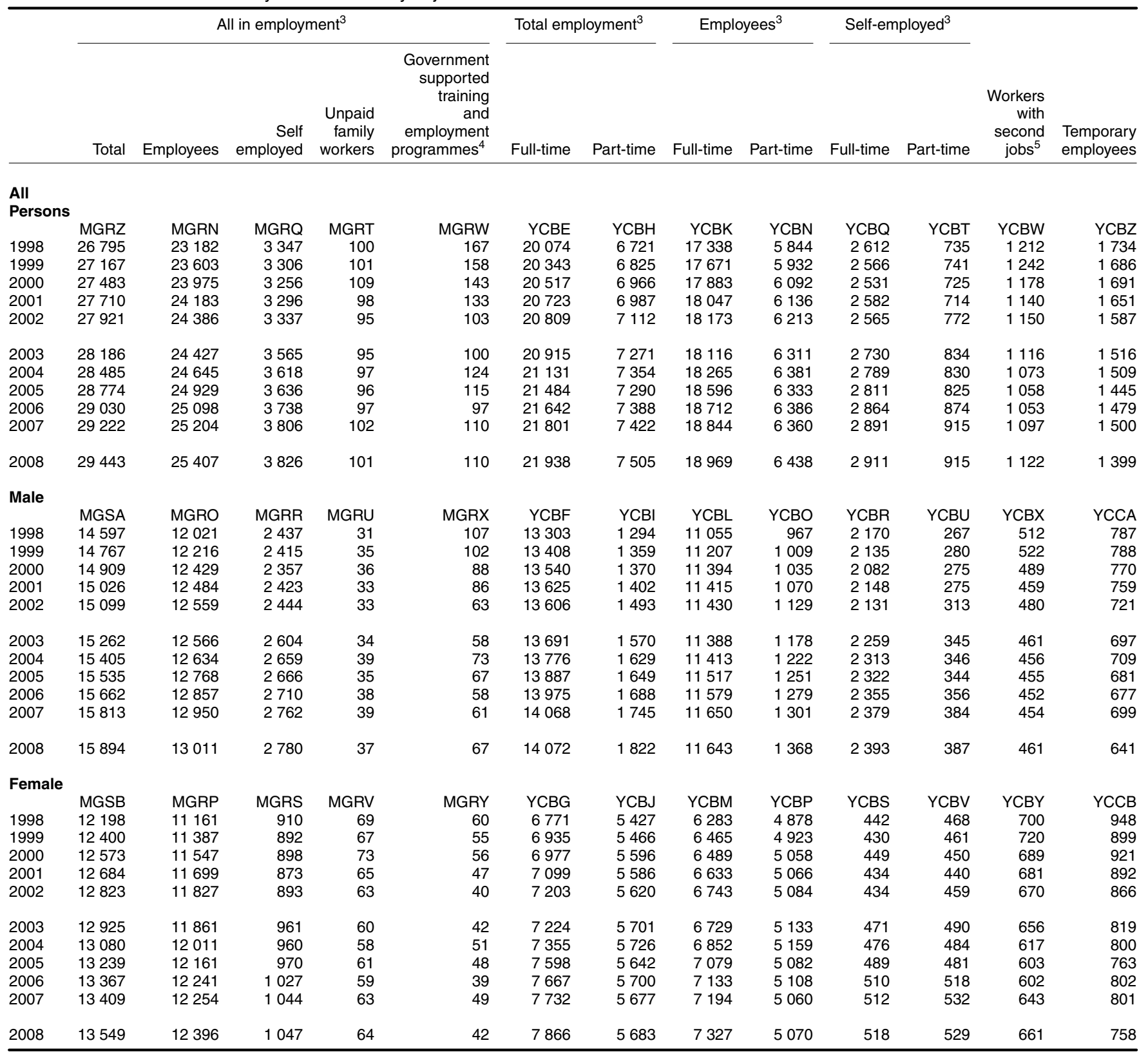

1 See chapter text. In August 2007, ONS published the mid-year population estimates for 2006. These estimates have now been incorporated into the LFS estimates from 2001 onwards. Further details can be found at http://www.statistics.gov.uk/cci/article.asp?id=1919

2 The Labour Force Survey has now moved to calendar quarters from May 2006. More information can be found on page 5 of the Concepts and Definitions.pdf by following this link:- www.statistics.gov.uk/downloads/theme labour/Concepts Definitions HQS.pdf

3 People whose main job is full or part-time and based on respondents' self assessment.

4 Those on employment and training programmes are classified as in employment. Some of those on programmes may consider themselves to be employees or self employed so appear in other categories.

5 Second jobs reported in LFS in addition to person's main full or part-time job. 


\section{Thousands}

All

Persons

2002

2003

2004

2005

2006

2007

2008

Male

2002

2003

2004

2005

2006

2007

2008

Female

2002

2003

2004

2005

2006

2007

2008

Percentages $^{3}$

All

\section{Persons}

2002

2003

2004

2005

2006

2007

2008

Male

2002

2003

2004

2005

2007

2008

Female

2002

2003

2004
2005

2006

2007

2008

1 See chapter text. In August 2007, ONS published the mid-year population estimates for 2006. These estimates have now been incorporated into the LFS estimates from 2001 onwards. Further details can be found at http://www.statistics.gov.uk/cci/article.asp?id=1919

2 The Labour Force Survey has now moved to calendar quarters from May

2006. More information can be found on page 5 of the Concepts and Defi-

nitions.pdf by following this link:- www.statistics.gov.uk/down-

loads/theme_labour/Concepts_Definitions HQS.pdf

3 Total in employment as a percentage of all persons in the relevant group.

$\begin{array}{rrr}\text { MGST } & \text { MGSW } & \text { YBU } \\ 53.0 & 69.6 & 45 \\ 53.1 & 69.7 & 45.1 \\ 53.5 & 69.9 & 43.9 \\ 53.7 & 70.0 & 41.0 \\ 53.8 & 70.0 & 39.0\end{array}$

\begin{tabular}{|c|c|c|c|c|c|}
\hline YBTO & YBTR & YBTU & YBTX & MGUW & MGUZ \\
\hline 659 & 3381 & 6509 & 10430 & 6055 & 887 \\
\hline 652 & 3415 & 6362 & 10591 & 6221 & 946 \\
\hline 642 & 3528 & 6283 & 10742 & 6293 & 998 \\
\hline 607 & 3539 & 6297 & 10885 & 6379 & 1070 \\
\hline 559 & 3618 & 6261 & 10975 & 6451 & 1167 \\
\hline 534 & 3657 & 6254 & 11039 & 6513 & 1226 \\
\hline 525 & 3663 & 6285 & 11065 & 6583 & 1323 \\
\hline YBTP & YBTS & YBTV & YBTY & MGUX & MGVA \\
\hline 321 & 1773 & 3566 & 5559 & 3582 & 297 \\
\hline 316 & 1802 & 3473 & 5659 & 3682 & 330 \\
\hline 309 & 1868 & 3417 & 5743 & 3731 & 337 \\
\hline 293 & 1879 & 3432 & 5781 & 3790 & 361 \\
\hline 260 & 1912 & 3423 & 5845 & 3832 & 390 \\
\hline 257 & 1943 & 3443 & 5879 & 3885 & 407 \\
\hline 260 & 1935 & 3444 & 5871 & 3943 & 443 \\
\hline YBTQ & YBTT & YBTW & YBTZ & MGUY & MGVB \\
\hline 338 & 1608 & 2943 & 4871 & 2473 & 591 \\
\hline 336 & 1612 & 2889 & 4932 & 2539 & 616 \\
\hline 333 & 1660 & 2866 & 4998 & 2562 & 661 \\
\hline 315 & 1660 & 2865 & 5104 & 2589 & 709 \\
\hline 299 & 1706 & 2838 & 5130 & 2619 & 776 \\
\hline 277 & 1714 & 2812 & 5160 & 2627 & 820 \\
\hline 265 & 1729 & 2841 & 5195 & 2640 & 880 \\
\hline
\end{tabular}

$\begin{array}{rrrrrr}\text { MGSR } & \text { MGSU } & \text { YBUA } & \text { YBUD } & \text { YBUG } & \text { YBUJ } \\ 59.7 & 74.5 & 43.6 & 67.5 & 79.6 & 81.9 \\ 59.9 & 74.6 & 42.7 & 66.5 & 79.5 & 82.1 \\ 60.0 & 74.7 & 41.2 & 66.9 & 79.7 & 82.2 \\ 60.1 & 74.7 & 38.6 & 65.6 & 80.2 & 82.4 \\ 60.2 & 74.6 & 35.5 & 65.5 & 80.1 & 82.3\end{array}$

YBUM

68.2
69.6

70.0

70.4

70.8

YBUP

8.5

8.9

9.4
9.9

10.7

71.3

11.0

80.3
80.3

82.3
82.4

71.9

11.7

YBUH YBUK

$88.0 \quad 88.3$

$\begin{array}{ll}87.6 & 88.7\end{array}$

$87.4 \quad 89.0$

$88.1 \quad 88.5$

88.7

YBUN

70.3

71.6

72.0

72.4

70.1

68.8
68.3

88.7

88.8

72.3

72.6
73.0

67.6

87.9

88.6

9.9
10.5

$\begin{array}{rrr}\text { YBUF } & \text { YBUI } & \text { YBUL } \\ 64.3 & 71.3 & 75.7 \\ 63.1 & 71.6 & 75.6 \\ 63.7 & 72.1 & 75.6 \\ 62.3 & 72.3 & 76.4 \\ 62.8 & 72.2 & 76.1\end{array}$

YBUO

YBUR

$\begin{array}{rrr} & \text { YBUO } & \text { YBUR } \\ 75.7 & 65.4 & 8.9 \\ 75.6 & 66.8 & 9.3\end{array}$

$\begin{array}{lll}75.6 & 66.8 & 9.3\end{array}$

$\begin{array}{lll}75.6 & 67.2 & 9.9\end{array}$

$\begin{array}{lll}76.4 & 67.8 & 10.5\end{array}$

$\begin{array}{ll}67.8 & 10.5 \\ 68.5 & 11.3\end{array}$

69.6

11.8

62.0
61.5

75.9

70.2

12.4

\begin{tabular}{llll}
72.8 & 76.3 & 70.2 & 12.4 \\
\hline
\end{tabular}
Helpline: 01633456901 


\begin{tabular}{|c|c|c|c|c|c|c|c|c|c|c|c|c|}
\hline & & 1998 & 1999 & 2000 & 2001 & 2002 & 2003 & 2004 & 2005 & 2006 & 2007 & 2008 \\
\hline \multicolumn{13}{|l|}{ United Kingdom } \\
\hline Claimant count & BCJD & 1347.8 & 1248.1 & 1088.4 & 969.9 & 946.6 & 933.0 & 853.3 & 862.1 & 944.7 & 863.3 & 902.4 \\
\hline Males & DPAE & 1029.4 & 955.0 & 831.6 & 739.6 & 717.1 & 700.3 & 636.2 & 639.7 & 697.3 & 630.6 & 663.3 \\
\hline Females & DPAF & 318.4 & 293.1 & 256.8 & 230.3 & 229.6 & 232.8 & 217.1 & 222.1 & 247.4 & 232.7 & 239.1 \\
\hline Workforce jobs & DYDC & 28824 & 29127 & 29554 & 29890 & 30064 & 30350 & 30671 & 31012 & 31257 & 31471 & 31661 \\
\hline Males & KAMS & 15327 & 15663 & 15772 & 15992 & 16002 & 16245 & 16376 & 16487 & 16662 & 16773 & 16908 \\
\hline Females & KAMT & 13497 & 13464 & 13782 & 13898 & 14061 & 14105 & 14296 & 14525 & 14596 & 14698 & 14753 \\
\hline Males & KAMV & 203 & 201 & 199 & 196 & 197 & 203 & 199 & 191 & 185 & 180 & 176 \\
\hline Females & KAMW & 17 & 17 & 18 & 18 & 18 & 19 & 19 & 18 & 18 & 18 & 18 \\
\hline Self-employment jobs & DYZN & 3691 & 3688 & 3579 & 3604 & 3674 & 3883 & 3964 & 3943 & 4056 & 4169 & 4181 \\
\hline Males & KAMZ & 2639 & 2642 & 2552 & 2593 & 2637 & 2785 & 2863 & 2840 & 2879 & 2953 & 2961 \\
\hline Females & KANA & 1052 & 1046 & 1027 & 1012 & 1037 & 1097 & 1101 & 1103 & 1177 & 1216 & 1220 \\
\hline Employees jobs & BCAJ & 24783 & 25091 & 25639 & 25973 & 26085 & 26152 & 26381 & 26763 & 26933 & 27051 & 27232 \\
\hline Males & KANC & 12409 & 12740 & 12948 & 13142 & 13114 & 13201 & 13249 & 13398 & 13559 & 13609 & 13739 \\
\hline $\begin{array}{l}\text { Females } \\
\text { of whom }\end{array}$ & KAND & 12374 & 12351 & 12691 & 12831 & 12971 & 12951 & 13132 & 13365 & 13374 & 13442 & 13493 \\
\hline Total, all manufacturing & & & & & & & & & & & & \\
\hline industries & KANG & 4208 & 4059 & 3959 & 3805 & 3599 & 3410 & 3246 & 3102 & 2975 & 2911 & 2867 \\
\hline Government-supported trainees & KANH & 131 & 131 & 119 & 99 & 90 & 92 & 108 & 96 & 65 & 53 & 54 \\
\hline Males & KANI & 76 & 81 & 73 & 62 & 55 & 55 & 65 & 58 & 38 & 31 & 32 \\
\hline Females & KANJ & 55 & 50 & 46 & 38 & 36 & 37 & 44 & 38 & 27 & 21 & 22 \\
\hline \multicolumn{13}{|l|}{ Great Britain } \\
\hline Claimant count & DPAG & 1290.3 & 1197.3 & 1046.3 & 930.5 & 910.2 & 898.5 & 822.5 & 833.2 & 916.8 & 839.0 & 874.7 \\
\hline Males & ZSDP & 984.6 & 915.7 & 799.6 & 709.7 & 689.3 & 673.9 & 612.8 & 618.0 & 676.4 & 612.3 & 641.8 \\
\hline Females & ZSDQ & 305.7 & 281.6 & 246.8 & 220.8 & 220.9 & 224.6 & 209.8 & 215.1 & 240.4 & 226.7 & 232.9 \\
\hline Workforce jobs & KANQ & 28103 & 28394 & 28804 & 29127 & 29289 & 29561 & 29870 & 30186 & 30423 & 30632 & 30810 \\
\hline Males & KANR & 14937 & 15267 & 15366 & 15580 & 15588 & 15822 & 15946 & 16044 & 16214 & 16327 & 16454 \\
\hline Females & KANS & 13167 & 13126 & 13438 & 13547 & 13702 & 13739 & 13924 & 14142 & 14209 & 14305 & 14355 \\
\hline HM Forces & $\mathrm{BCAH}$ & 219 & 218 & 217 & 214 & 214 & 223 & 218 & 210 & 204 & 198 & 193 \\
\hline Males & KANU & 203 & 201 & 199 & 196 & 197 & 203 & 199 & 191 & 185 & 180 & 176 \\
\hline Females & KANV & 17 & 17 & 18 & 18 & 18 & 19 & 19 & 18 & 18 & 18 & 18 \\
\hline Self-employment jobs & KANW & 3598 & 3592 & 3480 & 3500 & 3572 & 3774 & 3851 & 3820 & 3933 & 4055 & 4059 \\
\hline Employee jobs & KANZ & 24169 & 24465 & 24997 & 25321 & 25419 & 25478 & 25699 & 26067 & 26227 & 26332 & 26507 \\
\hline Males & KAOA & 12105 & 12429 & 12630 & 12821 & 12788 & 12873 & 12917 & 13062 & 13215 & 13258 & 13386 \\
\hline Females & KAOB & 12065 & 12036 & 12367 & 12500 & 12631 & 12605 & 12782 & 13005 & 13012 & 13074 & 13120 \\
\hline \multirow{2}{*}{\multicolumn{13}{|c|}{$\begin{array}{l}\text { of whom } \\
\text { Total, production and }\end{array}$}} \\
\hline & & & & & & & & & & & & \\
\hline construction industries & KAOC & 5383 & 5239 & 5205 & 5052 & 4813 & 4616 & 4464 & 4344 & 4290 & 4239 & 4242 \\
\hline \multicolumn{13}{|l|}{ Total, all manufacturing } \\
\hline industries & KAOD & 4101 & 3954 & 3856 & 3704 & 3501 & 3318 & 3157 & 3015 & 2888 & 2823 & 2778 \\
\hline Government-supported trainees & KAOE & 117 & 120 & 110 & 92 & 84 & 86 & 102 & 90 & 58 & 46 & 51 \\
\hline Males & KAOF & 67 & 73 & 67 & 57 & 50 & 51 & 60 & 53 & 33 & 27 & 29 \\
\hline Females & KAOG & 50 & 47 & 43 & 35 & 34 & 35 & 42 & 36 & 25 & 19 & 21 \\
\hline
\end{tabular}

Note. Because the figures have been rounded independently totals may differ from the sum of the components. Also the totals may include some employees whose industrial classification could not be ascertained.

Sources: Employment, Earnings and Innovations Division, Office for National

1 The data in this table have not been adjusted to reflect the 2001 Census population data. See chapter text.

2 There is a discontinuity in the employee jobs series between December 2005 and September 2006 due to improvements to the annual benchmark.

Further information can be found at:

http://www.statistics.gov.uk/statbase/product.asp?vlnk=9765 


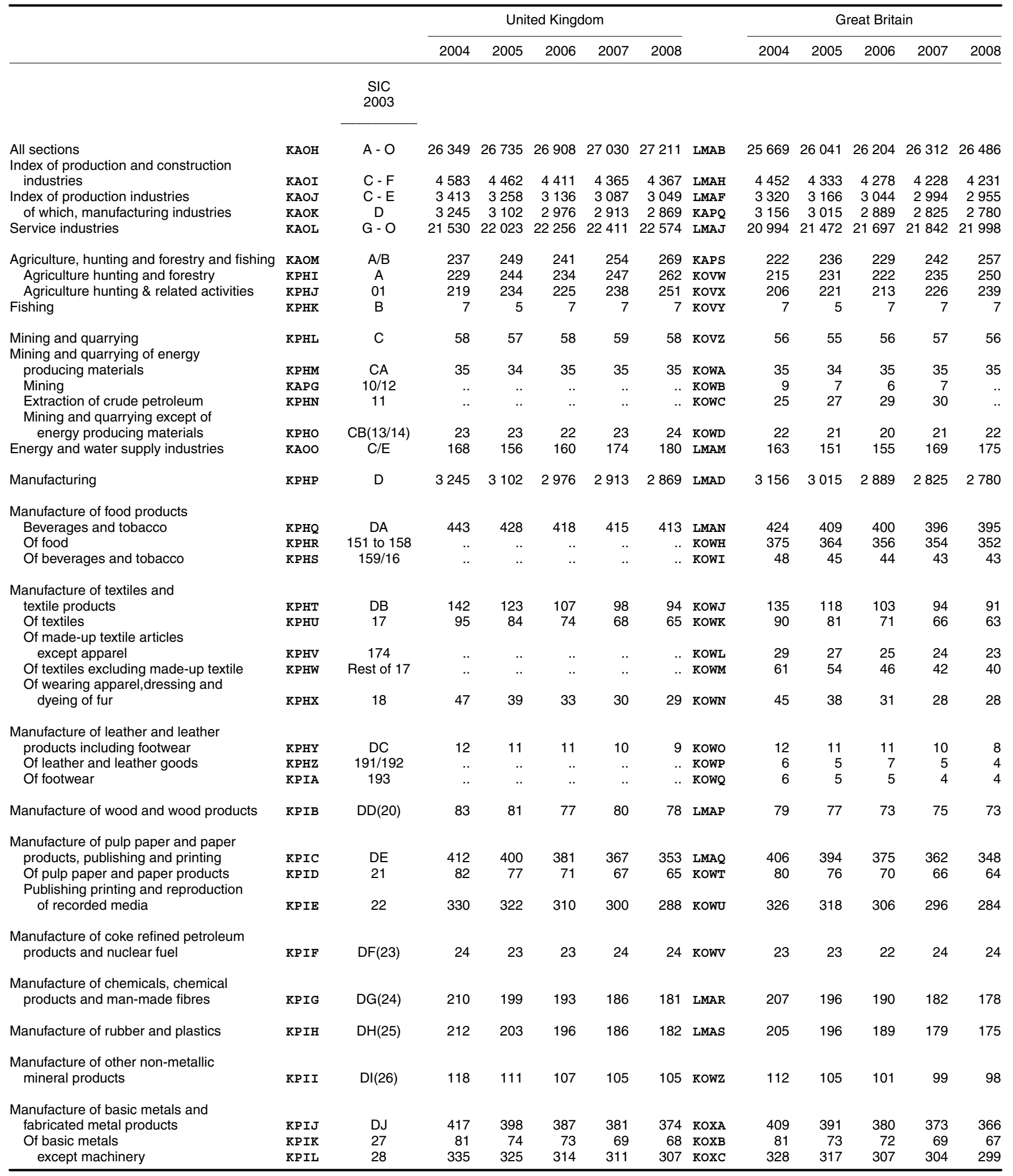




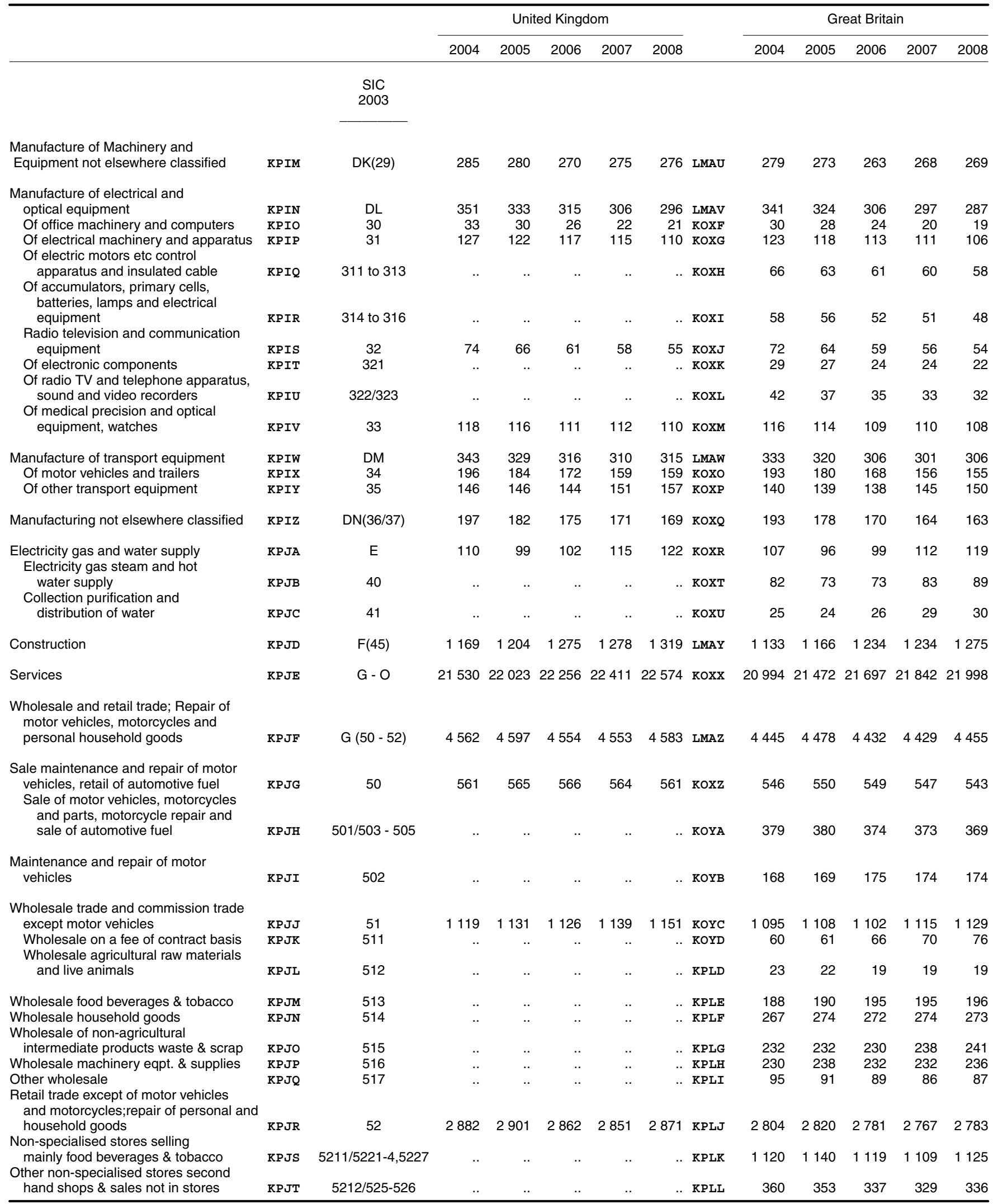




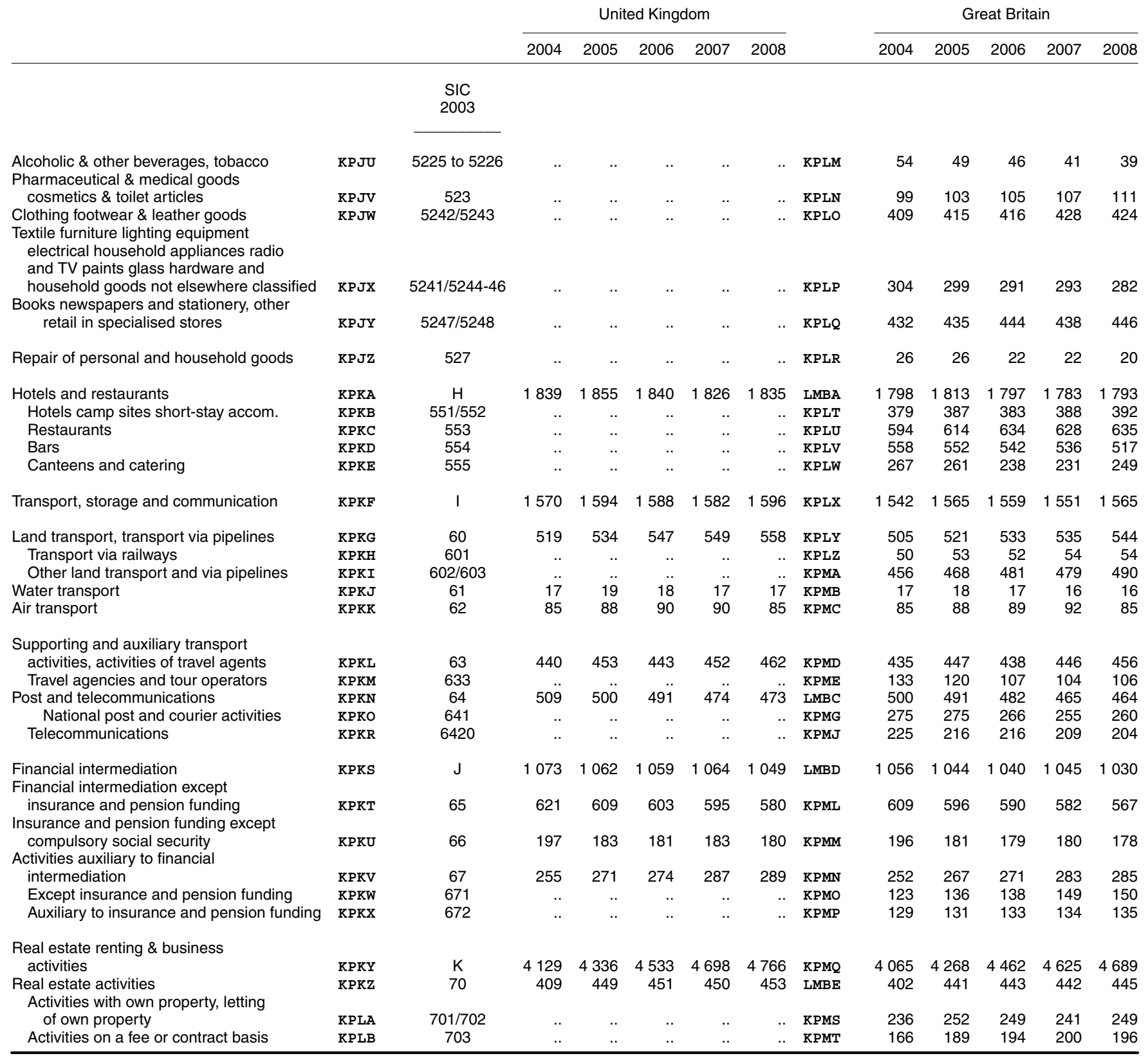


\begin{tabular}{c} 
United Kingdom \\
\hline $2004 \quad 2005 \quad 2006 \quad 2007 \quad 2008$
\end{tabular}

$20042005 \quad 2006 \quad 2007 \quad 2008$ $2004 \quad 2005 \quad 2006 \quad 2007 \quad 2008$ employees whose industrial classification could not be ascertained.

1 See chapter text. The data in this table have not been adjusted to reflect the 2001 Census population data.

2 All figures have been revised. For further information see: http://www. statistics.gov.uk/cci/article.asp?id=1340

3 There is a discontinuity in the employee jobs series between December 2005 and September 2006 due to improvements to the annual benchmark. Further information can be found at: http://www.statistics.gov.uk/Statbase/Product.asp?vink=9765

4 Head office and holding company local units were reclassified to Class 74.15 (within Section K) from December 2003 as a result of the SIC 2003 update.

\begin{tabular}{|c|c|c|c|c|c|c|c|c|c|c|c|c|c|}
\hline & & $\begin{array}{c}\text { SIC } \\
2003\end{array}$ & & & & & & & & & & & \\
\hline \multicolumn{14}{|l|}{ Renting of machinery and equipment without } \\
\hline $\begin{array}{l}\text { operator \& of personal \& household goods } \\
\text { Construction and civil engineering }\end{array}$ & KPLC & 71 & 153 & 157 & 160 & 157 & 156 & KPMU & 150 & 155 & 158 & 155 & 154 \\
\hline machinery & KOUU & 7132 & .. & .. & .. & .. & .. & KPMV & 41 & 42 & 45 & 45 & 46 \\
\hline All other goods and equipment & KoUV & Rest of 71 & .. & .. & .. & .. & .. & KPMW & 109 & 113 & 113 & 110 & 108 \\
\hline Computer and related equipment & KOUW & 72 & 488 & 493 & 525 & 542 & 548 & KPMX & 482 & 486 & 518 & 535 & 540 \\
\hline Research and development & KOUX & 73 & 101 & 104 & 108 & 109 & 108 & KPMY & 100 & 103 & 106 & 108 & 106 \\
\hline Other business activities & KOUY & 74 & 2978 & 3133 & 3289 & 3440 & 3501 & KPMZ & 2930 & 3083 & 3238 & 3386 & 3444 \\
\hline Legal, accounting, book-keeping \& auditing activities & KOUZ & 741 & .. & .. &. & .. &.. & KPNA & 840 & 889 & 962 & 1005 & 1027 \\
\hline Legal activities & KOVA & 7411 & .. & .. & .. & .. & .. & KPNB & 254 & 260 & 280 & 294 & 292 \\
\hline \multicolumn{14}{|l|}{ Accounting, book-keeping auditing, } \\
\hline tax consultancy & KOVB & 7412 & .. & .. & .. & .. &.. & KPNC & 194 & 203 & 218 & 226 & 238 \\
\hline consultancy activities & Kovc & $7413 / 7414$ & .. & .. & .. & .. & .. & KPND & 291 & 316 & 355 & 377 & 389 \\
\hline Management activities of holding companies ${ }^{4}$ & KOVD & 7415 & .. & .. & .. & .. & .. & KPNE & 101 & 110 & 109 & 108 & 108 \\
\hline \multicolumn{14}{|l|}{ Architectural engineering activities } \\
\hline technical testing & KOVE & $742 / 743$ & .. & .. & .. & .. & .. & KPNF & 340 & 347 & 374 & 394 & 410 \\
\hline Advertising & KOVF & 744 & .. & .. & .. & .. & .. & KPNG & 80 & 84 & 81 & 87 & 83 \\
\hline Industrial cleaning & KOVG & 747 & .. & .. & .. & .. & .. & KPNH & 414 & 436 & 449 & 449 & 455 \\
\hline Public administration and defence, & KOVH & $\mathrm{L}(75)$ & 1492 & 1516 & 1514 & 1509 & 1474 & LMBG & 1431 & 1456 & 1454 & 1450 & 1415 \\
\hline Education & KOVI & $\mathrm{M}(80)$ & 2299 & 2348 & 2384 & 2401 & 2420 & LMBH & 2230 & 2274 & 2311 & 2328 & 2348 \\
\hline Health and social work & KOVJ & $\mathrm{N}$ & 3178 & 3298 & 3345 & 3364 & 3421 & LOJV & 3070 & 3188 & 3233 & 3249 & 3305 \\
\hline Human health, veterinary activities & KOVK & $851 / 852$ & .. & .. & .. & .. & .. & KPNL & 2027 & 2103 & 2100 & 2095 & 2123 \\
\hline Social work activities & KOVL & 853 & .. & .. & .. & .. & .. & KPNM & 1043 & 1084 & 1133 & 1153 & 1182 \\
\hline \multicolumn{14}{|l|}{$\begin{array}{l}\text { Other community social and personal } \\
\text { service activities, private households } \\
\text { with employed persons, extra-territorial }\end{array}$} \\
\hline organisations and bodies & KOVM & $\mathrm{O}$ & 1388 & 1417 & 1440 & 1414 & 1431 & LMBK & 1357 & 1385 & 1408 & 1382 & 1398 \\
\hline Sewage and refuse disposal; sanitation & KOVN & 90 & 100 & 104 & 108 & 110 & 109 & KPNO & 97 & 101 & 105 & 106 & 106 \\
\hline Activities of membership organisations & kovo & 91 & 212 & 215 & 225 & 215 & 208 & KPNP & 204 & 206 & 217 & 207 & 200 \\
\hline $\begin{array}{l}\text { Recreational cultural and sporting activities } \\
\text { Motion picture video radio TV news }\end{array}$ & KOVP & 92 & 762 & 779 & 783 & 771 & 789 & KPNQ & 747 & 764 & 767 & 755 & 773 \\
\hline $\begin{array}{l}\text { agencies and entertainment activities } \\
\text { Other service activities, private }\end{array}$ & KovQ & 921 to 924 & .. & .. & .. & .. &.. & KPNR & 224 & 222 & 222 & 218 & 232 \\
\hline $\begin{array}{l}\text { households with employed persons, } \\
\text { extra territorial organisations }\end{array}$ & KOVT & 93/95/99 & 314 & 318 & 325 & 319 & 325 & KPNU & 309 & 313 & 320 & 314 & 320 \\
\hline Washing, dry cleaning of textile & & & & & & & & & & & & & \\
\hline $\begin{array}{l}\text { and fur products } \\
\text { Hairdressing, other beauty treatment, }\end{array}$ & KOVU & 9301 & .. & .. & .. & .. & .. & KPNV & 42 & 40 & 43 & 40 & 38 \\
\hline physical and well-being activities & Kovv & $9302 / 9304$ & .. & .. & .. & .. & .. & KPNW & 105 & 114 & 122 & 122 & 122 \\
\hline
\end{tabular}

Note. Because the figures have been rounded independently totals may differ from the sum of the components. Also the totals may include some

Renting of machinery and equipment without machinery

Legal activities

consultancy activities

Architectural engineering activities

technical testing

Advertising

ublic administration and defence,

Education

Health and social work

Social work activities

Sources: Department of Manpower Services (Northern Ireland) Employment, Earnings and Innovations Division, ONS: 01633456776 


\section{Weekly hours worked: by $\operatorname{sex}^{1,2}$ \\ At Quarter 2 each year ${ }^{3}$. Seasonally adjusted}

Hours

All workers' weekly hours ${ }^{4,5}$

Total (millions)

Average

Average actual weekly hours of work ${ }^{5}$

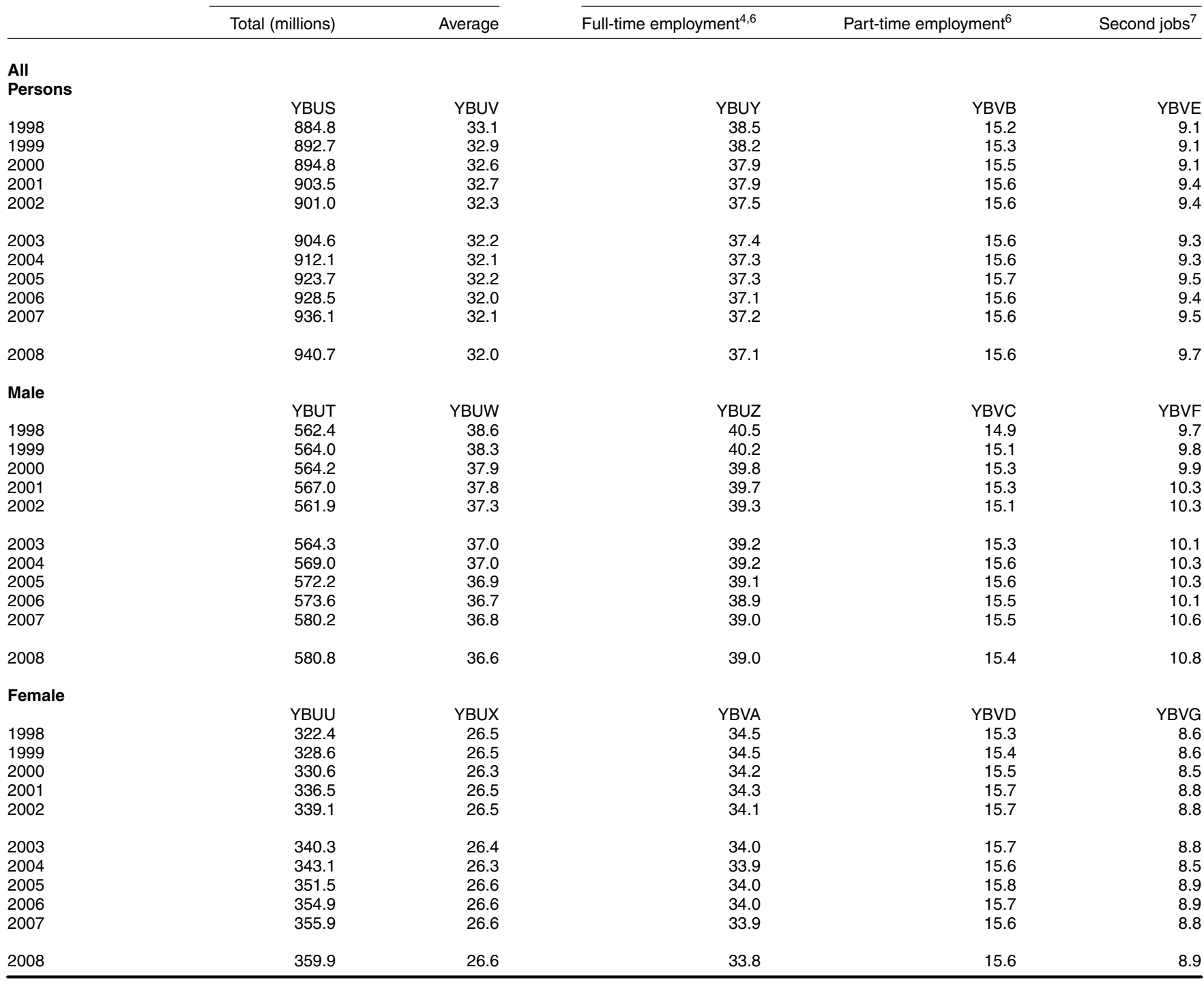

1 See chapter text. In August 2007, ONS published the mid-year population 6 People whose main job is full-time or part-time and based on respondents' self estimates for 2006. These estimates have now been incorporated into the assessment.

LFS estimates from 2001 onwards. Further details can be found at 7 Second jobs reported in the LFS in addition to persons' main full time job. http://www.statistics.gov.uk/cci/article.asp?id=1919

2 Average hours actually worked in the reference week which includes hours worked in second jobs.

Sources: Labour Force Survey, Office for National Statistics

3 The Labour Force Survey has now moved to calendar quarters from May

2006. More information can be found on page 5 of the Concepts and Defi-

nitions.pdf by following this link:- www.statistics.gov.uk/down-

loads/theme_labour/Concepts_Definitions_HQS.pdf

4 Main and second job.

5 Includes both paid and unpaid overtime. 


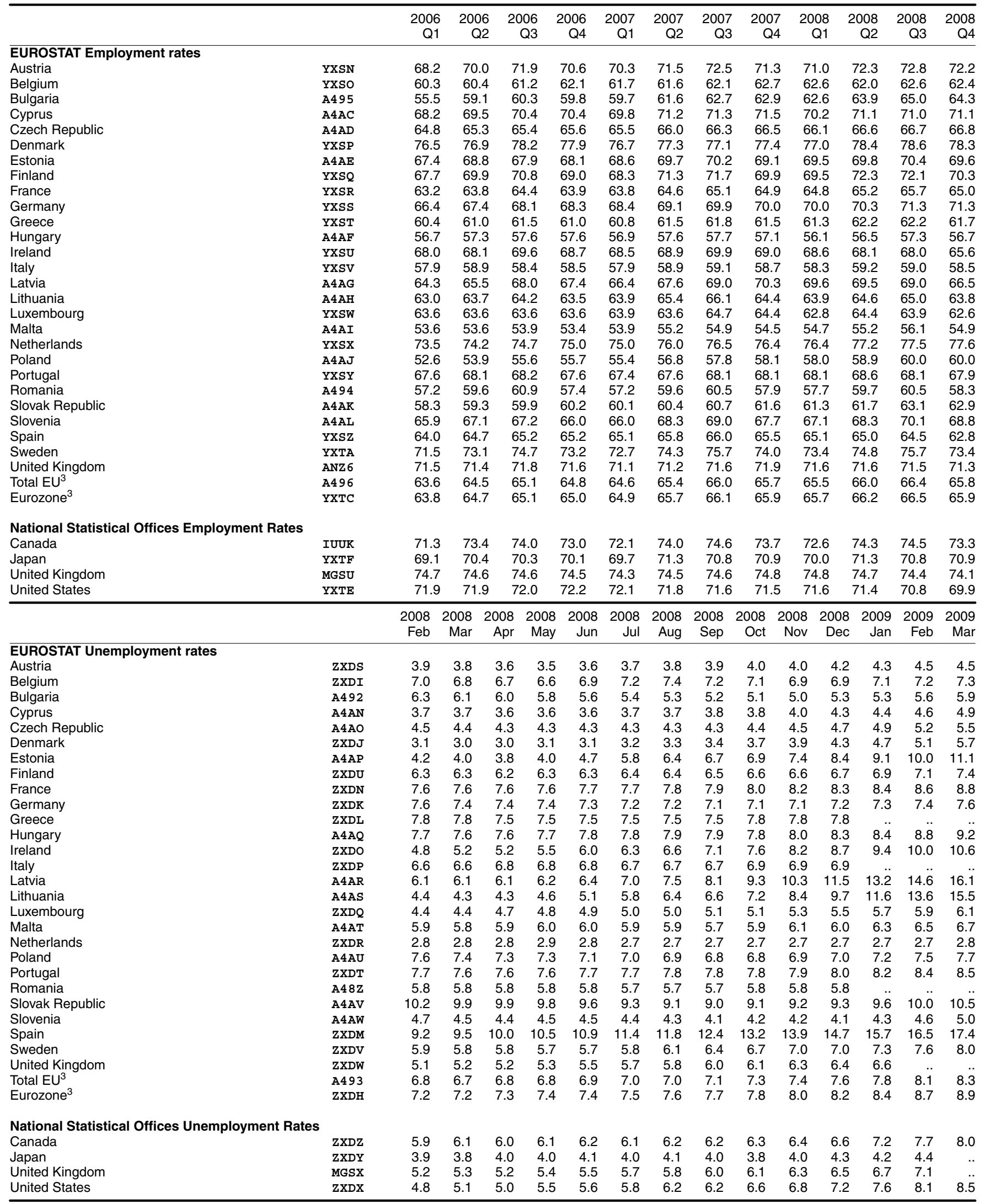

1 See chapter text.

3 The "Total EU" series consists of all 27 EU countries. The Eurozone series

2 The UK employment rate as published by the Office for National Statistics is consists of the following EU countries: Austria, Belgium, Cyprus, Finland, seasonally adjusted. All other employment and unemployment rates are not France, Germany, Greece, Ireland, Italy, Luxembourg, Malta, Netherlands, Porseasonally adjusted. 
Labour Market

Full-time equivalents, not seasonally adjusted

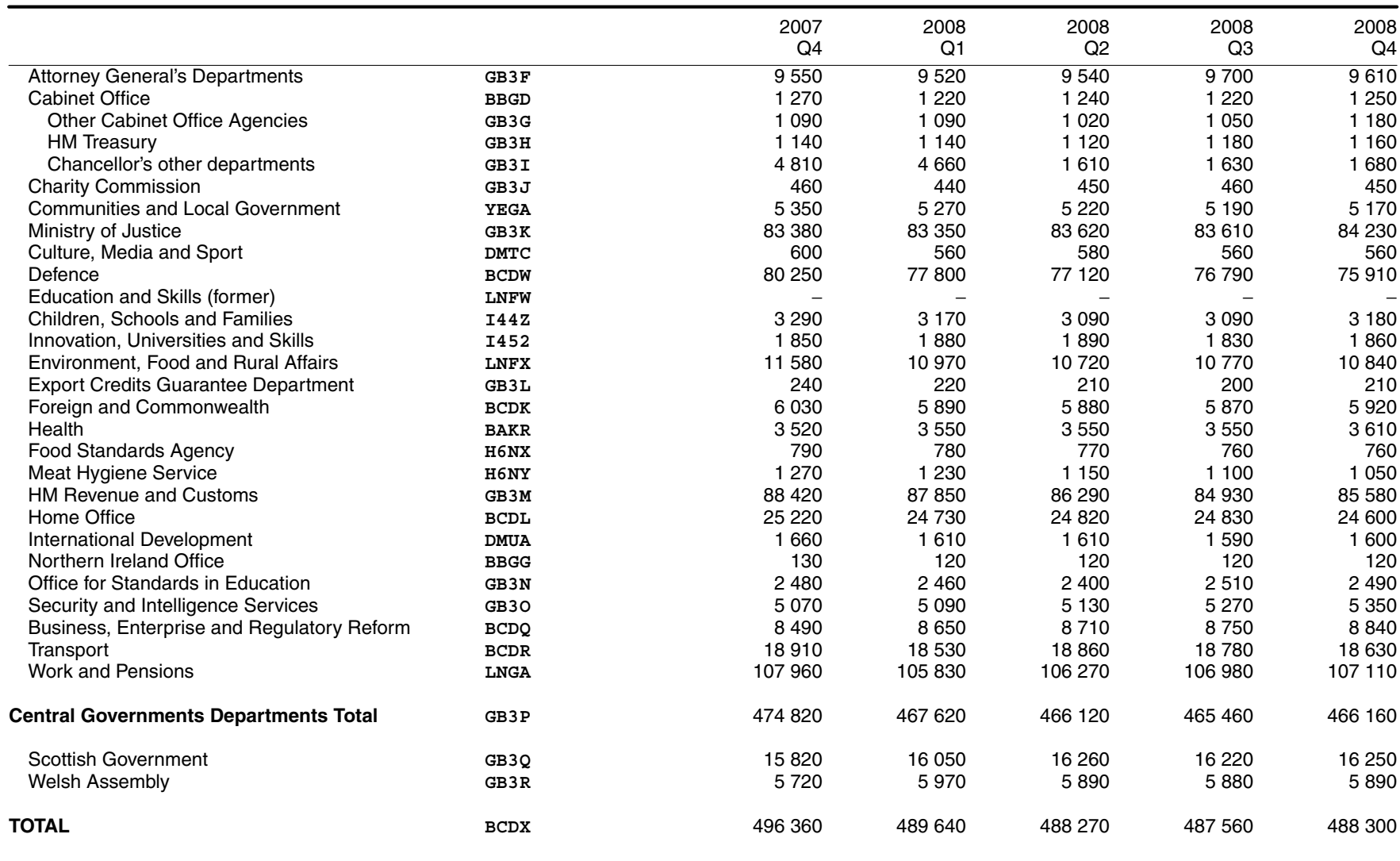

All

\section{Persons}

2002

2003

2004

2005

2006

MGSC
1521
1468
1439
1438
1687
1662
1685

YBSH
1499
1450
1422
1421
1661

1633
1662

\section{YBVH}

162 YBVN

176

173

177
179

179

2007
2008

Male

2002

2003

2004

2005

2006

2007

2008

Female

2002

2003

2004

2005

2006

2007

2008

$\begin{array}{rr}\text { MGSD } & \text { YBSI } \\ 914 & 905 \\ 890 & 880 \\ 845 & 837 \\ 837 & 829 \\ 978 & 967 \\ & \\ 950 & 937 \\ 990 & 980\end{array}$

196
188

388
400
402
432
514

517
526

$\begin{array}{rr}\text { YBVI } & \text { YBVO } \\ 93 & 243 \\ 99 & 244 \\ 99 & 231 \\ 101 & 261 \\ 105 & 309 \\ & \\ 109 & 312 \\ 102 & 331\end{array}$

YCGM

342

318
299

299
285

341

YCGS

387

343

353
338

419

305
327

\section{3}

405

MGVL

221

213

195

190
208

208

223

YCGN

193
182

182
185

160

\section{YCGT}

227
211

185

183

229

205

161
182

218

216

\section{0}

180

160
680

450
170

230

560

180

860

210

(20

760

1050
8580

600

600
120

490

840

18630

160

250

300

sis 


\begin{tabular}{|c|c|c|c|c|c|c|c|c|}
\hline & At Quarter 2 each year ${ }^{3}$. & Seasona & Isted & & & & \multicolumn{2}{|c|}{ Percentages } \\
\hline & $\begin{array}{r}\text { All aged } 16 \text { and } \\
\text { over }\end{array}$ & $16-59 / 64$ & $16-17$ & $18-24$ & $25-34$ & $35-49$ & $\begin{array}{l}50-64(\mathrm{~m}) \\
50-59(\mathrm{w})\end{array}$ & $\begin{array}{l}65+(m) \\
60+(w)\end{array}$ \\
\hline \multicolumn{9}{|c|}{ All Persons } \\
\hline & MGSX & YBTI & YBVK & YBVQ & YCGP & YCGV & MGXE & $\mathrm{MGXH}$ \\
\hline 2001 & 5.0 & 5.1 & 18.6 & 10.1 & 4.9 & 3.6 & 3.3 & 1.7 \\
\hline 2002 & 5.2 & 5.3 & 20.0 & 10.3 & 5.0 & 3.6 & 3.5 & 2.4 \\
\hline 2003 & 5.0 & 5.1 & 21.4 & 10.6 & 4.8 & 3.1 & 3.3 & 1.9 \\
\hline 2004 & 4.8 & 4.9 & 21.5 & 10.2 & 4.6 & 3.2 & 3.0 & 1.7 \\
\hline 2005 & 4.8 & 4.9 & 21.9 & 10.9 & 4.3 & 3.0 & 2.9 & 1.6 \\
\hline 2006 & 5.5 & 5.6 & 24.0 & 12.5 & 5.2 & 3.7 & 3.1 & 2.3 \\
\hline 2007 & 5.4 & 5.5 & 27.3 & 12.4 & 4.6 & 3.4 & 3.3 & 2.3 \\
\hline 2008 & 5.4 & 5.6 & 25.9 & 12.5 & 4.9 & 3.5 & 2.9 & 1.7 \\
\hline \multicolumn{9}{|l|}{ Male } \\
\hline & MGSY & YBTJ & YBVL & YBVR & YCGQ & YCGW & MGXF & MGXI \\
\hline 2001 & 5.6 & 5.6 & 21.6 & 11.2 & 5.3 & 3.7 & 4.0 & \\
\hline 2002 & 5.7 & 5.8 & 22.3 & 12.1 & 5.1 & 3.9 & 4.0 & 3.2 \\
\hline 2003 & 5.5 & 5.6 & 23.6 & 12.0 & 4.9 & 3.6 & 3.8 & .. \\
\hline 2004 & 5.2 & 5.3 & 24.4 & 11.0 & 5.2 & 3.1 & 3.6 & .. \\
\hline 2005 & 5.1 & 5.2 & 24.8 & 12.2 & 4.5 & 3.1 & 3.2 & .. \\
\hline 2006 & 5.9 & 6.0 & 28.3 & 14.1 & 5.3 & 3.8 & 3.4 & 2.7 \\
\hline 2007 & 5.7 & 5.7 & 30.9 & 13.8 & 4.5 & 3.4 & 3.7 & 3.2 \\
\hline 2008 & 5.8 & 5.9 & 28.2 & 14.5 & 5.0 & 3.6 & 3.5 & 2.3 \\
\hline \multicolumn{9}{|c|}{ Female } \\
\hline & MGSZ & YBTK & YBVM & YBVS & YCGR & YCGX & MGXG & MGXJ \\
\hline 2001 & 4.4 & 4.6 & 15.6 & 8.9 & 4.3 & 3.5 & 2.2 & .. \\
\hline 2002 & 4.5 & 4.6 & 17.6 & 8.2 & 4.8 & 3.2 & 2.8 & 2.1 \\
\hline 2003 & 4.3 & 4.4 & 19.0 & 8.9 & 4.5 & 2.6 & 2.6 & .. \\
\hline 2004 & 4.3 & 4.5 & 18.6 & 9.4 & 3.8 & 3.3 & 2.2 & .. \\
\hline 2005 & 4.4 & 4.5 & 19.0 & 9.4 & 4.2 & 2.9 & 2.4 & .. \\
\hline 2006 & 5.0 & 5.2 & 19.7 & 10.7 & 5.0 & 3.6 & 2.7 & 2.0 \\
\hline 2007 & 5.1 & 5.3 & 23.8 & 10.8 & 4.9 & 3.5 & 2.7 & 1.8 \\
\hline 2008 & 4.9 & 5.1 & 23.6 & 10.2 & 4.9 & 3.5 & 2.3 & 1.4 \\
\hline
\end{tabular}

Note: Where figure denoted as .. it is not shown as it is based on a small sample size and is subject to a margin of uncertainty.

Sources: Labour Force Survey, Office for National Statistics;

1 See chapter text. In August 2007, ONS published the mid-year population Helpline: 01633456901 estimates for 2006. These estimates have now been incorporated into the LFS estimates from 2001 onwards. Further details can be found at http://www.statistics.gov.uk/cci/article. asp?id=1919

2 Total unemployment as a percentage of all economically active persons in the relevant age group.

3 The Labour Force Survey has now moved to calendar quarters from May 2006. More information can be found on page 5 of the Concepts and Definitions.pdf by following this link:- www.statistics.gov.uk/downloads/theme_labour/Concepts_Definitions_HQS.pdf 
Labour Market

Duration of unemployment: by sex ${ }^{1,2}$

United Kingdom

At Quarter 2 each year ${ }^{3}$. Seasonally adjusted

Thousands

\begin{tabular}{|c|c|c|c|c|c|c|c|c|c|c|c|c|}
\hline & & 1998 & 1999 & 2000 & 2001 & 2002 & 2003 & 2004 & 2005 & 2006 & 2007 & 2008 \\
\hline \multicolumn{13}{|l|}{ All Persons } \\
\hline $\begin{array}{l}\text { All unemployed }{ }^{4} \\
\text { Duration of unemployment }\end{array}$ & MGSC & 1791 & 1743 & 1600 & 1470 & 1521 & 1468 & 1439 & 1438 & 1687 & 1662 & 1685 \\
\hline Less than 6 months & YBWF & 981 & 984 & 920 & 877 & 980 & 950 & 927 & 911 & 1025 & 1002 & 994 \\
\hline 6 months \& less than 1 year & YBWG & 253 & 268 & 240 & 218 & 219 & 206 & 228 & 215 & 302 & 264 & 276 \\
\hline 1 year or more & YBWH & 557 & 491 & 441 & 375 & 322 & 312 & 284 & 311 & 360 & 396 & 415 \\
\hline 1 year or more as $\%$ of total & YBWI & 31.1 & 28.2 & 27.5 & 25.5 & 21.2 & 21.3 & 19.8 & 21.7 & 21.3 & 23.8 & 24.6 \\
\hline All unemployed ${ }^{4}$ & MGSD & 1076 & 1060 & 959 & 882 & 914 & 890 & 845 & 837 & 978 & 950 & 990 \\
\hline \multicolumn{13}{|l|}{ Duration of unemployment } \\
\hline Less than 6 months & MGYK & 519 & 535 & 501 & 482 & 539 & 539 & 506 & 485 & 545 & 527 & 540 \\
\hline 6 months \& less than 1 year & MGYM & 160 & 166 & 140 & 132 & 147 & 132 & 141 & 138 & 177 & 155 & 162 \\
\hline 1 year or more & MGYO & 397 & 360 & 318 & 268 & 228 & 218 & 198 & 213 & 255 & 269 & 288 \\
\hline 1 year or more as $\%$ of total & YBWJ & 36.9 & 33.9 & 33.2 & 30.4 & 24.9 & 24.5 & 23.4 & 25.5 & 26.1 & 28.3 & 29.1 \\
\hline \multicolumn{13}{|l|}{ Female } \\
\hline All unemployed ${ }^{4}$ & MGSE & 715 & 683 & 641 & 589 & 607 & 579 & 593 & 601 & 709 & 712 & 695 \\
\hline 6 months \& less than 1 year & MGYN & 93 & 102 & 100 & 87 & 72 & 74 & 86 & 77 & 125 & 109 & 114 \\
\hline 1 year or more & MGYP & 160 & 131 & 122 & 107 & 94 & 94 & 87 & 98 & 105 & 127 & 127 \\
\hline 1 year or more as $\%$ of total & YBWK & 22.4 & 19.2 & 19.1 & 18.3 & 15.5 & 16.3 & 14.6 & 16.4 & 14.7 & 17.9 & 18.3 \\
\hline
\end{tabular}

1 All aged 16 and over. See chapter text.

Sources: Labour Force Survey, Office for National Statistics;

2 In August 2007, ONS published the mid-year population estimates for 2006. Helpline: 01633456901

These estimates have now been incorporated into the LFS estimates from

2001 onwards. Further details can be found at http://www.statistics.gov.uk/cci/article. asp?id=1919

3 The Labour Force Survey has now moved to calendar quarters from May 2006. More information can be found on page 5 of the Concepts and Definitions.pdf by following this link:- www.statistics.gov.uk/downloads/theme labour/Concepts Definitions HQS.pdf

4 Totals include people who did not state their duration of unemployment. 
Annual averages

Males

All ages

All durations

Up to 6 months

Over 6 and up to 12 months

All over 12 months

All over 24 months

AGNG

AGXK

ELNP

ELON

IKBS

Aged 18 to 24

All durations

Up to 6 months

Over 6 and up to 12 months

All over 12 months

All over 24 months

\section{Aged 25 to 49}

All durations

Up to 6 months

Over 6 and up to 12 months

All over 12 months

All over 24 months

Aged $\mathbf{5 0}$ and over

All durations

Up to 6 months

Over 6 and up to 12 months

All over 12 months

All over 24 months

\section{Females}

\section{All ages}

All durations

Up to 6 months

Over 6 and up to 12 months

All over 12 months

All over 24 months

Aged 18 to 24

All durations

Up to 6 months

Over 6 and up to 12 months

All over 12 months

All over 24 months

JLGC

JLGD

JLGE

JLGF

JLGH

AGMA

JLHG

JLHH

JLHI

JLHK

JLHL

JLHM

JLHN

JLHO

JLHQ

JLGI

JLGK

JLGJ

JLGL

JIGN

163.6
35.5

27.6

9.7

JLGO

JLGP

JLGO

JLGR

Aged 25 to 49

All durations

Up to 6 months

Over 6 and up to 12 months

All over 12 months

All over 24 months

Aged $\mathbf{5 0}$ and over

All durations

Up to 6 months

Over 6 and up to 12 months

All over 12 months

All over 24 months

1.8
0.2

JLHR

JLHS

JLHT

JLHU

76.7

18.5

16.2
457.4
124.2
126.7

126.7
50.7

168.1

141.0
23.8

0.3

421.8

254.9
80.9

86.1
29.2

118.4

61.6
19.6

37.3

21.1

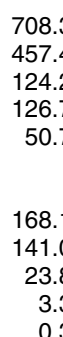

693.0

451.2

127.1

114.7

37.6

171.9

143.8

24.5
3.6

0.4

404.8

248.0

82.9

73.8

17.0

116.3

59.4

19.7
37.2

37.2

20.2

$$
\begin{array}{r}
630.7 \\
408.8 \\
113.7 \\
108.2 \\
34.6 \\
\\
\\
\\
161.8 \\
134.3 \\
23.3 \\
4.2 \\
0.5
\end{array}
$$

362.3

221.3

72.9
68.1

14.1

106.7

53.2

17.5

35.9

19.9
635.0
423.4
113.3
98.2
33.1


174.6
143.6
25.9
5.0
0.6

357.4

225.9

70.4

61.2
14.2

103.0

53.9
17.0

32.0

18.3

694.0
438.6
136.5
119.0
34.5


195.5
155.0
33.1
7.5
0.9

628.5

405.2

111.1

112.3

33.9

176.7
145.7

145.7
25.3

5.8

1.1

387.5

228.9

83.4

75.2

352.5

211.5

69.6

71.4

14.5

111.0

54.7

20.0

18.4

99.3

48.0

16.3

35.0

18.2

$\begin{array}{rrr}245.9 & 231.8 & 238.2 \\ 171.5 & 164.2 & 181.0 \\ 43.6 & 37.5 & 34.3 \\ 30.8 & 30.1 & 22.9 \\ 8.4 & 8.4 & 5.8\end{array}$

$75.0 \quad 77.3$

62.9
10.4
1.8

$\begin{array}{rr}230.1 & 214.7 \\ 166.3 & 153.2 \\ 37.2 & 34.9 \\ 26.5 & 26.7 \\ 8.1 & 8.0\end{array}$

220.0
159.2
35.6
25.2
7.9

8.4

$\begin{array}{rrrrrr}77.3 & 74.0 & 79.1 & 90.2 & 85.2 & 88.1 \\ 64.9 & 61.5 & 65.3 & 72.2 & 70.7 & 76.0 \\ 10.6 & 10.4 & 11.4 & 14.7 & 12.0 & 10.2 \\ 1.8 & 2.1 & 2.3 & 3.3 & 2.5 & 1.9 \\ 0.3 & 0.3 & 0.4 & 0.5 & 0.5 & 0.5\end{array}$

$111.4 \quad 112$

112.1
77.3

77.3
19.9

14.9

102.1

69.0

18.2

14.9

101.9

70.3
17.7

13.8

3.0

111.8

73.6
21.3

16.9

3.3

$105.1 \quad 109.5$

$69.3 \quad 77.5$

$\begin{array}{ll}18.8 & 17.7 \\ 17.0 & 14.3\end{array}$

$3.2 \quad 2.9$

$\begin{array}{lr}\text { JLHX } & 40 . \\ \text { JLHY } & 24 . \\ \text { JLHZ } & 6 . \\ \text { JLIA } & 9.7 \\ \text { JLIC } & 4.8\end{array}$

\begin{tabular}{rr}
40.7 & 38.7 \\
24.1 & 22.7 \\
6.8 & 6.3 \\
9.8 & 9.7 \\
4.7 & 4.8 \\
\hline
\end{tabular}

\begin{tabular}{rrrrr}
38.7 & 39.1 & 44.0 & 41.4 & 40.6 \\
22.7 & 23.5 & 25.6 & 24.1 & 27.5 \\
6.3 & 6.5 & 7.8 & 6.8 & 6.4 \\
9.7 & 9.1 & 10.6 & 10.6 & 6.7 \\
4.8 & 4.5 & 4.7 & 4.7 & 2.4 \\
\hline
\end{tabular}

1 Count of claimants of unemployment-related benefits.

Source: Office for National Statistics: 01633456901 
At Quarter 2 each year ${ }^{4}$. Seasonally adjusted ${ }^{5}$

Percentages

\begin{tabular}{|c|c|c|c|c|c|c|c|c|c|c|c|c|}
\hline & & 1998 & 1999 & 2000 & 2001 & 2002 & 2003 & 2004 & 2005 & 2006 & 2007 & 2008 \\
\hline North East & YCNC & 8.3 & 9.6 & 8.9 & 7.5 & 6.5 & 6.1 & 5.5 & 6.8 & 6.1 & 6.3 & 7.5 \\
\hline North West & YCND & 6.9 & 6.3 & 5.3 & 5.3 & 5.6 & 5.0 & 4.5 & 4.4 & 5.3 & 5.8 & 6.3 \\
\hline Yorkshire and The Humber & $\mathrm{YCNE}$ & 7.3 & 6.3 & 6.1 & 5.5 & 5.3 & 5.1 & 4.6 & 4.8 & 5.8 & 5.5 & 6.1 \\
\hline East Midlands & YCNF & 4.9 & 5.3 & 4.8 & 5.0 & 4.6 & 4.3 & 4.3 & 4.3 & 5.5 & 5.0 & 5.7 \\
\hline West Midlands & YCNG & 5.9 & 7.0 & 6.1 & 5.5 & 5.7 & 5.6 & 5.5 & 4.6 & 5.6 & 6.7 & 6.3 \\
\hline East & $\mathrm{YCNH}$ & 4.9 & 4.3 & 3.6 & 3.6 & 3.7 & 3.9 & 3.8 & 3.9 & 5.0 & 4.6 & 4.6 \\
\hline South East & YCNJ & 4.4 & 3.9 & 3.3 & 3.2 & 3.9 & 4.0 & 3.7 & 3.8 & 4.7 & 4.3 & 4.2 \\
\hline South West & YCNK & 4.8 & 4.5 & 4.3 & 3.5 & 3.7 & 3.4 & 3.7 & 3.2 & 3.8 & 4.0 & 3.8 \\
\hline Wales & YCNM & 7.0 & 7.5 & 6.1 & 6.1 & 5.7 & 4.5 & 4.2 & 4.5 & 5.6 & 5.5 & 4.9 \\
\hline Scotland & YCNN & 7.4 & 7.1 & 7.0 & 6.2 & 6.3 & 5.3 & 6.0 & 5.5 & 5.5 & 4.7 & 4.2 \\
\hline Northern Ireland & ZSFB & 6.9 & 7.6 & 6.7 & 6.1 & 5.6 & 5.2 & 5.2 & 5.0 & 4.3 & 3.8 & 4.2 \\
\hline
\end{tabular}

1 Total unemployed as a percentage of all economically active persons.

Sources: Labour Force Survey, Office for National Statistics;

2 All aged 16 and over. See chapter text.

Helpline: 01633456901

3 In August 2007, ONS published the mid-year population estimates for 2006

These estimates have now been incorporated into the LFS estimates from 2001 onwards. Further details can be found at http://www.statistics.gov.uk/cci/article.asp?id=1919

4 The Labour Force Survey has now moved to calendar quarters from May 2006. More information can be found on page 5 of the Concepts and Definitions.pdf by following this link:- www.statistics.gov.uk/downloads/theme_labour/Concepts_Definitions_HQS.pdf

5 Previously not seasonally adjusted data was shown.

\section{Claimant count rates: by region ${ }^{1}$ \\ Seasonally adjusted annual averages}

\begin{tabular}{|c|c|c|c|c|c|c|c|c|c|c|c|c|}
\hline & & 1998 & 1999 & 2000 & 2001 & 2002 & 2003 & 2004 & 2005 & 2006 & 2007 & 2008 \\
\hline United Kingdom & BCJE & 4.5 & 4.1 & 3.6 & 3.1 & 3.1 & 3.0 & 2.7 & 2.7 & 2.9 & 2.7 & 2.8 \\
\hline North East & DPDM & 7.0 & 7.0 & 6.2 & 5.6 & 5.1 & 4.5 & 4.0 & 3.9 & 4.1 & 4.0 & 4.3 \\
\hline North West & IBWC & 5.0 & 4.6 & 4.1 & 3.7 & 3.5 & 3.3 & 2.9 & 2.9 & 3.3 & 3.1 & 3.4 \\
\hline Yorkshire and the Humber & DPBI & 5.4 & 5.0 & 4.3 & 3.9 & 3.6 & 3.3 & 2.8 & 2.9 & 3.3 & 3.0 & 3.3 \\
\hline East Midlands & DPBJ & 3.9 & 3.6 & 3.3 & 3.1 & 2.9 & 2.8 & 2.5 & 2.5 & 2.8 & 2.6 & 2.8 \\
\hline West Midlands & DPBN & 4.5 & 4.4 & 4.0 & 3.7 & 3.5 & 3.5 & 3.3 & 3.4 & 3.9 & 3.7 & 3.8 \\
\hline London & DPDQ & 5.1 & 4.4 & 3.7 & 3.3 & 3.5 & 3.6 & 3.5 & 3.4 & 3.4 & 3.0 & 2.9 \\
\hline South East & DPDR & 2.6 & 2.3 & 1.8 & 1.5 & 1.6 & 1.7 & 1.6 & 1.6 & 1.8 & 1.6 & 1.7 \\
\hline South West & DPBM & 3.4 & 3.0 & 2.5 & 2.0 & 1.9 & 1.9 & 1.6 & 1.6 & 1.8 & 1.6 & 1.7 \\
\hline England & VASQ & 4.3 & 3.9 & 3.4 & 3.0 & 2.9 & 2.9 & 2.6 & 2.6 & 2.9 & 2.6 & 2.8 \\
\hline Wales & DPBP & 5.4 & 5.0 & 4.4 & 3.9 & 3.5 & 3.3 & 3.0 & 3.0 & 3.1 & 2.8 & 3.1 \\
\hline Scotland & DPBQ & 5.3 & 5.0 & 4.5 & 3.9 & 3.8 & 3.7 & 3.4 & 3.2 & 3.3 & 2.8 & 2.8 \\
\hline Northern Ireland & DPBR & 7.3 & 6.3 & 5.3 & 4.9 & 4.4 & 4.2 & 3.6 & 3.3 & 3.2 & 2.8 & 3.2 \\
\hline
\end{tabular}

1 The number of unemployment-related benefit claimants as a percentage of

the estimated total workforce (the sum of claimants, employee jobs, self-

Source: Office for National Statistics: 01633456901

employed, participants on work-related government training programmes

and HM Forces) at mid-year. Excluded are claimants under 18, consistent

with current coverage. See chapter text. 


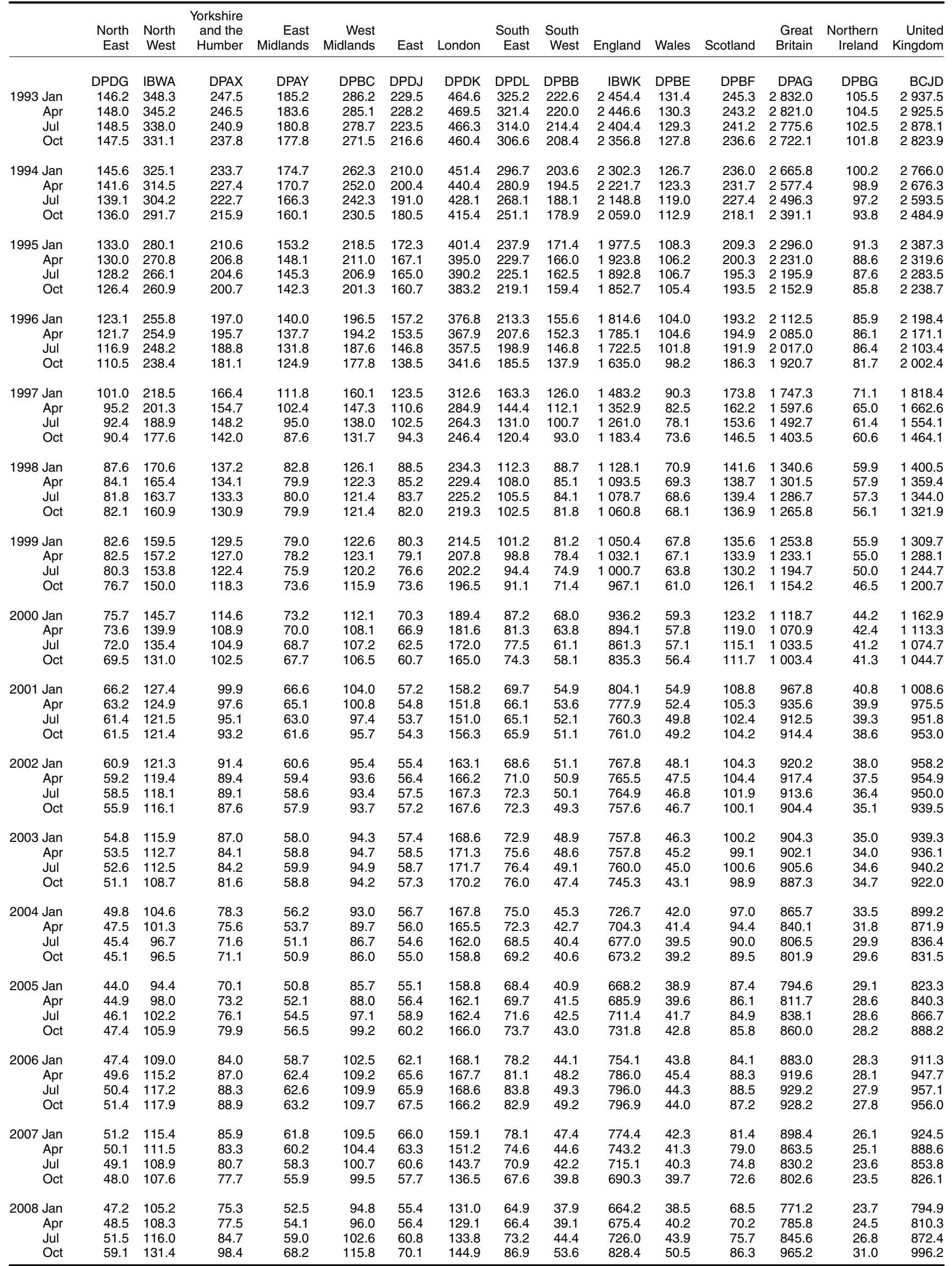
$\begin{array}{ll}1 \text { The figures are based on the number of claimants receiving unemployment } & \text { The latest national and regional seasonally adjusted claimant count figures are } \\ \text { related benefits and are adjusted for seasonality and discontinuities to be } & \text { provisional and subject to revision in the following month. }\end{array}$ 
Thousands

\section{All Persons}

2002

2003

2004

2005

2006

2007

2008

Male

2002

2003

2004

2007

2008

Female

2002

2003

2004

2005
2006

2007

2008

Percentages

All Persons

2002

2003

2004

2005

2006

2007

2008

Mal

2002

2003

2004

2005

2007

2008

Female

2002

2003

2004

2006

2007

2008

MGSF
29433
29659
29867
30170
30686
30821
31190

YBSK
28528
28712
28838
29095
29507
29586
29844

$\begin{array}{rr}\text { YBZL } & \text { YBZO } \\ 810 & 3777 \\ 826 & 3787 \\ 805 & 3927 \\ 807 & 3969 \\ 744 & 4109 \\ 718 & 4156 \\ 729 & 4202\end{array}$

YBSL

MGSG

15988

16187

16217

16352

15685
15848

15869

15991

16206

16742

16928

16329

16472

MGSH

13445

13472

13650

13819

14080

14079

14262

YBSM

12843

12864

12969

13104

13301

13257

13372

YBZM

416
418

418
405

407

370

354

363

YBZN

394

408

400

400
374

364

365

YBZP

2011
2035

2108

2139

2196

2252

2274

YBZQ

1766

1752

1819

1830
1913

1904

1927

BZO
777
987
927
109
156
202


$B Z P$
011
035
108
139
196
252
274


109
176
952
927
819
913

YBZR
6870
6687
6581
6569
6616
6562
6621

YBZU

10821

10947
11061

11201

11375

11429

11498

YBZS

3768

3674

3591

3581

3611

3611

3638

YBZT

3103

3013

2989

2988
3005

2950

2983
YBZV
5780
5869
5908
5965
6056
6087
6103

YBZW

5042

5078

5152

5236

5319

5342

5396

YCAM

83.8

83.4

83.4

83.6

84.6

84.3

84.8

YCAP

85.1
84.9

84.8

84.9

84.9
85.4

85.2

85.6

YCAN

92.7

91.9

91.9

93.0

YCAQ
92.0

92.1
91.7

91.4

92.0

93.1

91.9

$83.6 \quad 43.4$

70.8

83.7

43.4
44.5

78.5
77.6

93.1

92.1

YCAR
78.4
78.0
78.1
78.5
78.9

78.6
79.2

YCAO

YCAI YCAL

MGSQ

MGWI
55.6

55.5

55.8

56.1

72.9

55.0

52.7

52.1

73.3

49.0

68.8

70.0

68.8
70.5

74.5

75.1

75.4

47.2

74.1

47.2

69.0

56.7

76.5

MGWP

70.5

72.4

71.9

72.4

73.1

73.6

74.3

MGWS

8.6

9.0

10.0
10.8

11.1

11.9

1 See chapter text. In August 2007, ONS published the mid-year population estimates for 2006. These estimates have now been incorporated into the LFS estimates from 2001 onwards. Further details can be found at http://www.statistics.gov.uk/cci/article.asp?id=1919

2 The Labour Force Survey has now moved to calendar quarters from May 2006. More information can be found on page 5 of the Concepts and Definitions.pdf by following this link:- www.statistics.gov.uk/downloads/theme_labour/Concepts_Definitions_HQS.pdf

3 Total economically active as a percentage of all persons in the relevant age group.

Sources: Labour Force Survey, Office for National Statistics; Helpline: 01633456901 
Thousands

\section{All Persons}

$\begin{array}{lc}\text { All Persons } & \text { MG } \\ 2002 & 17317 \\ 2003 & 1738 \\ 2004 & 17524 \\ 2005 & 1765 \\ 2006 & 17531 \\ & \\ 2007 & 1780 \\ 2008 & 1781 \\ & \\ \text { Male } & \end{array}$

MGSI
7317
7 382

$-17382$

17653

17531

$$
\begin{aligned}
& \text { YBSN } \\
& 7751 \\
& 7769 \\
& 7892 \\
& 7960 \\
& 7836
\end{aligned}
$$

$\begin{array}{rr}\text { YCAS } & \text { YCAV } \\ 700 & 1212 \\ 699 & 1333 \\ 752 & 1330 \\ 766 & 1413 \\ 825 & 1396 \\ & \\ 869 & 1474 \\ 862 & 1534\end{array}$

YCAY
1330
1332
1306
1291
1205
1222
1183

YCBB
1891
1941
1984
1997
1952
1988
1939

MGWA

2619

2521

2494

2458

2002

2003

2004

2007

2008

MGSJ
6591
6562
6731
6835
6804
6899
6934

YBSO

3026

2987

YCAT

3116

3185

359
365

393

398

YCAW

486
538

538
549

585

595

YCAZ

295

299
317

315

273

3203

462

617

3200

267

269

2405

2355

\section{MGWD}

9566

9632

9693

9695

Female

2002

MGSK

10726

YBSP

10793

10818

10728

4725
4782

2004

2005
2006

2007

10901

4776

4775
4683

454

657

YCAU

341
334

334

359
368

390

4754

407

YCAX

725

795

780

828
800

857

857
877

YCBA

1035

1033

988
976

976
932

YCBC

YCBC
504

504
537

560

530

536

525

9842
9944

2008

10882

4672

955

914

YCBD
1387

1387

1436

1447

1437

1422

1452

1414

$\begin{array}{rr}\text { MGWB } & \text { MGWE } \\ 1381 & 3565 \\ 1280 & 3575 \\ 1320 & 3615 \\ 1328 & 3651 \\ 1319 & 3651 \\ & \\ 1323 & 3695 \\ 1297 & 3734\end{array}$

3734

MGWC

1237
1184

1184

1201

1166

1139

1083

1058

MGWF

6002

6017

6017
6043

6044

6147

Percentages $^{3}$

All Persons

YBTC

2002

2003

2004

2005

2006

2007

2008

Male

2002

2003

2004

2005
2006

2007

2008

Female

2002

2003

2004

2005

2006

2007

2008

$\begin{array}{rrr}\text { YBTL } & \text { LWEX } & \text { LWFA } \\ 21.4 & 46.3 & 24.3 \\ 21.3 & 45.8 & 26.0 \\ 21.5 & 48.3 & 25.3 \\ 21.5 & 48.7 & 26.3 \\ 21.0 & 52.6 & 25.4 \\ & & \\ 21.2 & 54.8 & 26 \\ 20.9 & 54.2 & 26.7\end{array}$

WFA
24.3

24.3
26.0

25.3

26.3

25.4

26.2
26.7

LWFD

16.2
16.6

16.6

16.6

16.4
15.4

LWFG

14.9
15.1

15.2

15.1

15.1
14.6

15.7

14.8

14.4

LWFJ

29.5

27.6

28.1

27.6

26.9

26.4

25.7

LWFM

91.4

91.0

90.4

90.0

89.2

88.9

88.1

$\begin{array}{rrrr}\text { YBTM } & \text { LWEY } & \text { LWFB } & \text { LWFE } \\ 16.2 & 46.3 & 19.5 & 7.3\end{array}$

19.5

20.7

21.5

21.3

LWFH

LWFK

LWFN

$\begin{array}{rrrr}7.3 & 8.0 & 27.1 & 92.2 \\ 7.5 & 7.9 & 24.9 & 91.3\end{array}$

$7.5 \quad 7.9$

8.3

24.9

25.5

25.4
24.9

91.3

91.2

91.0
90.1

$\begin{array}{ll}16.3 & 49.4\end{array}$

8.1
7.0

8.0

24.7

90.0

21.5
22.4

6.9

24.1

89.1

YBTE YBTN LWEZ LWFC LWFF

$\begin{array}{rr}\text { YBTE } & \text { YBTN } \\ 44.4 & 26.9\end{array}$

$44.5 \quad 27.1$

$44.2 \quad 26.9$

LWEZ

LWFC

31.2

30.0

31.2

47.3

47.9

31.2
29.5

LWFF
25.0

25.0
25.5

24.9

24.6

23.7

LWFI

$\begin{array}{r}21.6 \\ \hline\end{array}$

LWFL

LWFO

22.0
21.9

21.5

21.1

32.8

31.2

31.5

30.6

90.9

90.9

89.8

89.4

88.6

\begin{tabular}{ll}
31.0 & 24.5 \\
31.3 & 23.5 \\
\hline
\end{tabular}

21.4
20.8

28.6

88.2

Sources: Labour Force Survey, Office for National Statistics;

1 See chapter text. In August 2007, ONS published the mid-year population estimates for 2006. These estimates have now been incorporated into the LFS estimates from 2001 onwards. Further details can be found at http://www.statistics.gov.uk/cci/article. asp?id=1919

2 The Labour Force Survey has now moved to calendar quarters from May

2006. More information can be found on page 5 of the Concepts and Defi-

nitions.pdf by following this link:- www.statistics.gov.uk/down-

loads/theme_labour/Concepts_Definitions_HQS.pdf

3 Total economically inactive as a percentage of all persons in the relevant age group. 
Labour Market

\section{Economically inactive: ${ }^{1,2}$ by reason and sex \\ United Kingdom \\ At Quarter 2 each year ${ }^{3}$. Seasonally adjusted}

Thousands and percentages

\begin{tabular}{|c|c|c|c|c|c|c|c|c|c|}
\hline \multicolumn{7}{|c|}{ Economic inactivity by reason: } & \multicolumn{2}{|c|}{ by: } & \multirow{2}{*}{$\begin{array}{r}\text { All } \\
\text { economically } \\
\text { inactive }\end{array}$} \\
\hline Student & $\begin{array}{l}\text { Looking after } \\
\text { family/home }\end{array}$ & $\begin{array}{r}\text { Temporary } \\
\text { sick }\end{array}$ & $\begin{array}{r}\text { Long-term } \\
\text { sick }\end{array}$ & $\begin{array}{l}\text { Discouraged } \\
\text { workers }^{4}\end{array}$ & Retired & Other & $\begin{array}{r}\text { Does not want } \\
\text { a job }\end{array}$ & Wants a job & \\
\hline
\end{tabular}

Thousands

All Persons

2001 BEDZ

$2002 \quad 1516$

$2003 \quad 1655$

$2004 \quad 1712$

2005

1804

2343

1821

$\begin{array}{ll}2007 & 1920 \\ 2008 & 1969\end{array}$

2313
2348

2295

$\begin{array}{rr}\text { BEBK } & \text { BEBN } \\ 193 & 2209 \\ 179 & 2232 \\ 195 & 2109 \\ 193 & 2165 \\ 191 & 2145 \\ 191 & 2077 \\ 198 & 2034 \\ 181 & 1997\end{array}$

$\begin{array}{rrr}\text { YCFO } & \text { BEEI } & \text { BEEL } \\ 34 & 591 & 792 \\ 32 & 583 & 821 \\ 38 & 567 & 805 \\ 35 & 596 & 847 \\ 34 & 618 & 825 \\ & & \\ 37 & 584 & 813 \\ 34 & 609 & 813 \\ 39 & 590 & 801\end{array}$

YBVZ
5530
5499
5622
5864
5873
5750
5869
5709

YBWC

Male

$\begin{array}{rr} & \text { BEEX } \\ 2001 & 737\end{array}$

737 BEAQ

2002

2003

2004

2005

750

827

871
899

180

BEDI BEDL

$\begin{array}{ll}92 & 1240 \\ 88 & 1244 \\ 89 & 1149\end{array}$

1244
1149

1149
1180

187
192

1188

2006
2007

\section{9}

2008

993

195
207

$96 \quad 1137$

195

1137

88

1113

YCFP

BEDR BEDU

5709

2198
2252
2147
2028
2088
2086
2088
2163

YBSN

7751

7769

7892

7960

Female

BEBL BEBO

$\begin{array}{ll}777 & 2214 \\ 766 & 2205\end{array}$

2205

2222

$\begin{array}{ll}828 & 2222 \\ 842 & 2157\end{array}$

BEEG

102
91

BEEJ

970
988

959

985

905

2151

98

958

$\begin{array}{rrr}21 & 403 & 315 \\ 22 & 395 & 345 \\ 20 & 387 & 336\end{array}$

345
336

408

352
361

YBWA

2076
2085

2069

2255

2338

2163

7957

7872

2006

$912 \quad 2118$

$956 \quad 2141$

2100

940

897

22

\section{0}

432

342
357

2253

2316

2272

YBWD

$910 \quad$ YBSO

$941 \quad 3026$

$918 \quad 2987$

$861 \quad 3116$

$847 \quad 3185$

$\begin{array}{ll}900 & 3152\end{array}$

$\begin{array}{ll}887 & 3203 \\ 929 & 3200\end{array}$

2008

956
976

95
108
93

YCFQ BEEP

BEES
477
476
469
495
465

455
472
445

YBWB

3454

YBWE

1287
1311

1229

1167

1240

YBSP

3553

3609

3497

3553

1187

1201

4741
4725

4782

4776

4775

4683

3437

4754
4672

Percentages $^{5}$

$\begin{array}{lr}\text { All Persons } & \\ & \text { BED } \\ 2001 & 19 . \\ 2002 & 19.6 \\ 2003 & 21.3 \\ 2004 & 21.7 \\ 2005 & 22.7 \\ 2006 & 23 . \\ 2007 & 24 . \\ 2008 & 25 . \\ & \\ \text { Male } & \\ & \\ 2001 & \text { BEE } \\ 2002 & 24.7 \\ 2003 & 24.8 \\ 2004 & 27.7 \\ 2005 & 27.9 \\ & 28 . \\ 2006 & 28.8 \\ 2007 & 30.1 \\ 2008 & 31.0\end{array}$

$\begin{array}{rrr}\text { BEDM } & \text { BEDP } & \text { BEDS } \\ 31.0 & 2.5 & 28.6 \\ 30.8 & 2.3 & 28.8 \\ 30.9 & 2.5 & 27.1 \\ 29.7 & 2.4 & 27.4 \\ 29.4 & 2.4 & 26.9 \\ & & \\ 29.5 & 2.4 & 26.5 \\ 29.5 & 2.5 & 25.6 \\ 29.2 & 2.3 & 25.4\end{array}$

$\begin{array}{rrr}\text { BEDV } & \text { BEDY } & \text { BEEB } \\ 0.4 & 7.7 & 10.3 \\ 0.4 & 7.5 & 10.6 \\ 0.5 & 7.3 & 10.4 \\ 0.4 & 7.5 & 10.7 \\ 0.4 & 7.8 & 10.4 \\ & & \\ 0.5 & 7.5 & 10.4 \\ 0.4 & 7.7 & 10.2 \\ 0.5 & 7.5 & 10.2\end{array}$

$\begin{array}{rr}\text { BEEE } & \text { BEBM } \\ 71.6 & 28.4 \\ 70.9 & 29.1 \\ 72.4 & 27.6 \\ 74.3 & 25.7 \\ 73.8 & 26.2 \\ 73.4 & \\ 73.8 & 26.6 \\ 72.5 & 26.2 \\ & 27.5\end{array}$

BEAR

$\begin{array}{rrr}\text { BEEK } & \text { BEEN } & \text { BEEQ } \\ 6.0 & 3.1 & 41.5 \\ 6.0 & 2.9 & 41.1 \\ 6.0 & 3.0 & 38.5 \\ 6.0 & 3.1 & 37.9 \\ 6.0 & 3.2 & 37.3 \\ 6.2 & 3.0 & \\ 6.5 & 2.8 & 36.1 \\ 6.1 & 2.7 & 35.5 \\ & & 34.8\end{array}$

$\begin{array}{rrr}\text { BEET } & \text { BEEW } & \text { BEEZ } \\ 0.7 & 13.5 & 10.5 \\ 0.7 & 13.1 & 11.4 \\ 0.7 & 12.9 & 11.2 \\ 0.7 & 13.1 & 11.3 \\ 0.7 & 13.3 & 11.3 \\ & & \\ 0.9 & 13.6 & 11.4 \\ 0.6 & 13.9 & 10.7 \\ 0.7 & 13.5 & 11.2\end{array}$

$\begin{array}{rr}\text { BEAS } & \text { BEGT } \\ 69.5 & 30.5 \\ 68.9 & 31.1 \\ 69.3 & 30.7 \\ 72.4 & 27.6 \\ 73.4 & 26.6 \\ 71.5 & \\ 72.3 & 28.5 \\ 71.0 & 27.7\end{array}$

BEBP

100
100

100
100

100

$\begin{array}{ll}27.6 .6 & 100\end{array}$

\begin{tabular}{|c|c|c|c|c|c|c|c|c|c|c|}
\hline \multicolumn{11}{|c|}{ Female } \\
\hline & BEGZ & BEHC & BEHF & BEHI & BEHL & BEHO & BEBQ & BEHR & BEHU & BEGW \\
\hline 2001 & 16.4 & 46.7 & 2.1 & 20.4 & 0.3 & 4.0 & 10.1 & 72.8 & 27.2 & 100 \\
\hline 2002 & 16.2 & 46.7 & 1.9 & 20.9 & 0.2 & 4.0 & 10.1 & 72.3 & 27.7 & 100 \\
\hline 2003 & 17.3 & 46.5 & 2.2 & 20.1 & 0.4 & 3.8 & 9.8 & 74.3 & 25.7 & 100 \\
\hline 2005 & 18.9 & 45.0 & 1.9 & 20.1 & 0.3 & 4.1 & 9.7 & 74.0 & 26.0 & 100 \\
\hline 2006 & 19.5 & 45.2 & 2.0 & 20.1 & 0.2 & 3.3 & 9.7 & 74.7 & 25.3 & 100 \\
\hline 2007 & 20.1 & 45.0 & 2.3 & 18.9 & 0.3 & 3.5 & 9.9 & 74.7 & 25.3 & 100 \\
\hline 2008 & 20.9 & 44.9 & 2.0 & 18.9 & 0.4 & 3.4 & 9.5 & 73.6 & 26.4 & 100 \\
\hline
\end{tabular}

1 All persons aged 16-59 (women) / 16-64 (men). See chapter text.

Definitions.pdf by following this link:- www.statistics.gov.uk/downloads

2 In August 2007, ONS published the mid-year population estimates for /theme labour/Concepts Definitions HQS.pdf

2006. These estimates have now been incorporated into the LFS esti- 4 People whose reason for not seeking work was that they believed no jobs were mates from 2001 onwards. Further details can be found at available.

http://www.statistics.gov.uk/cci/article.asp?id=1919

5 Reasons for inactivity as a percentage of all economically inactive.

3 The Labour Force Survey has now moved to calendar quarters from May

2006. More information can be found on page 5 of the Concepts and

Sources: Labour Force Survey, Office for National Statistics;

Helpline: 01633456901 


\section{Labour Market}

\section{Labour disputes: by industry ${ }^{1}$ \\ - United Kingdom}

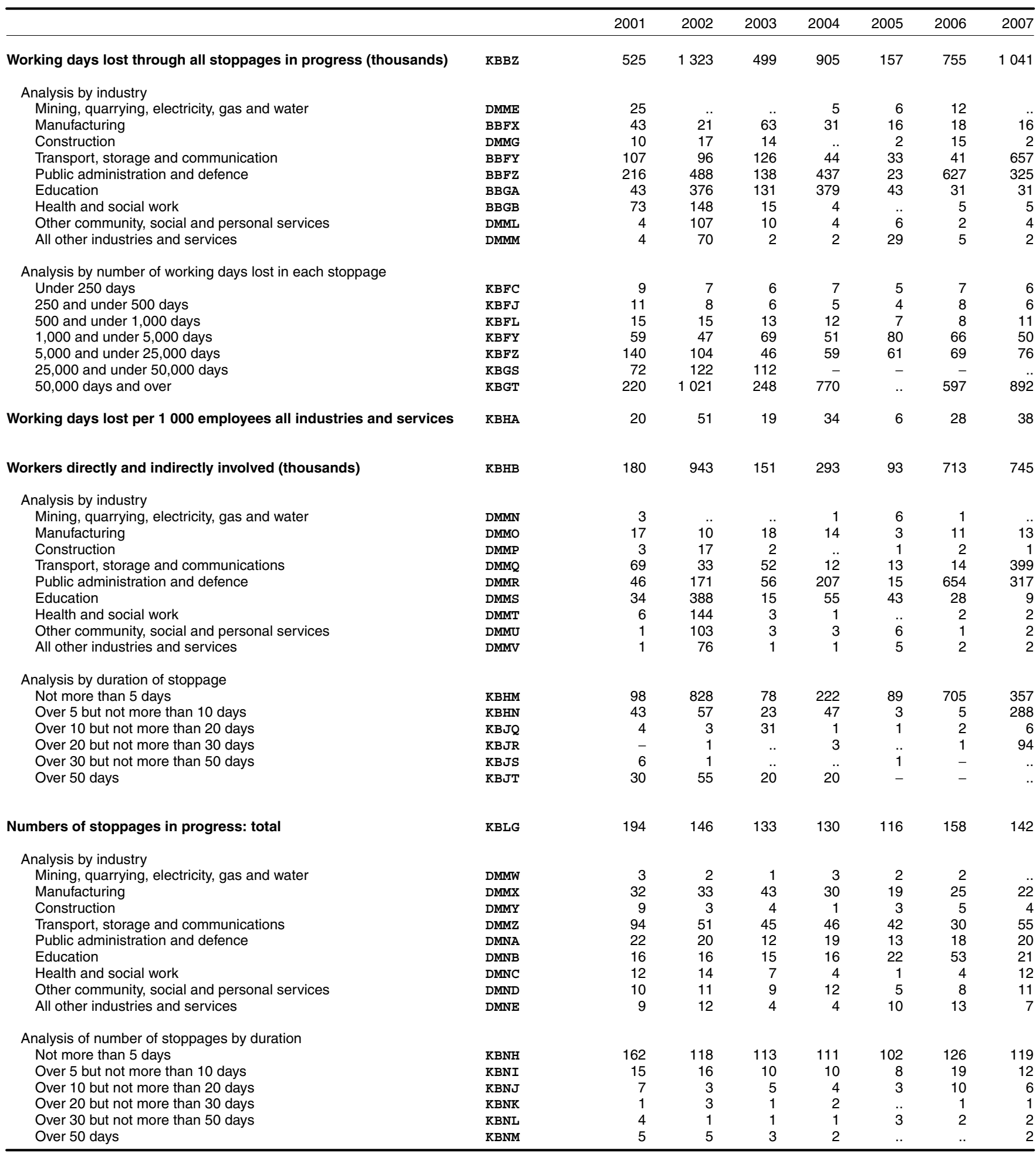

1 See chapter text.

Source: Labour Market Statistics, Office for National Statistics: 01633456721 
Labour Market

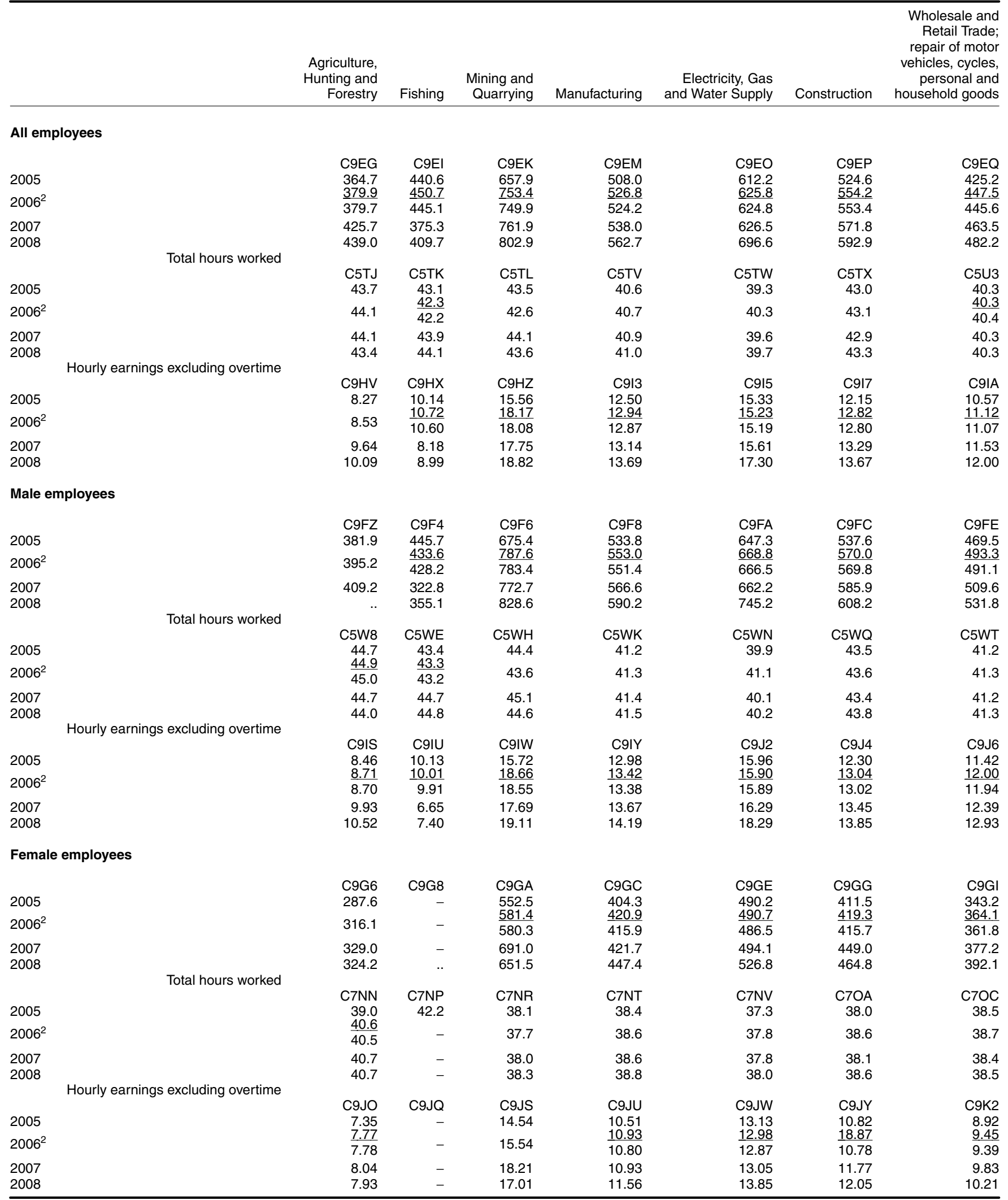




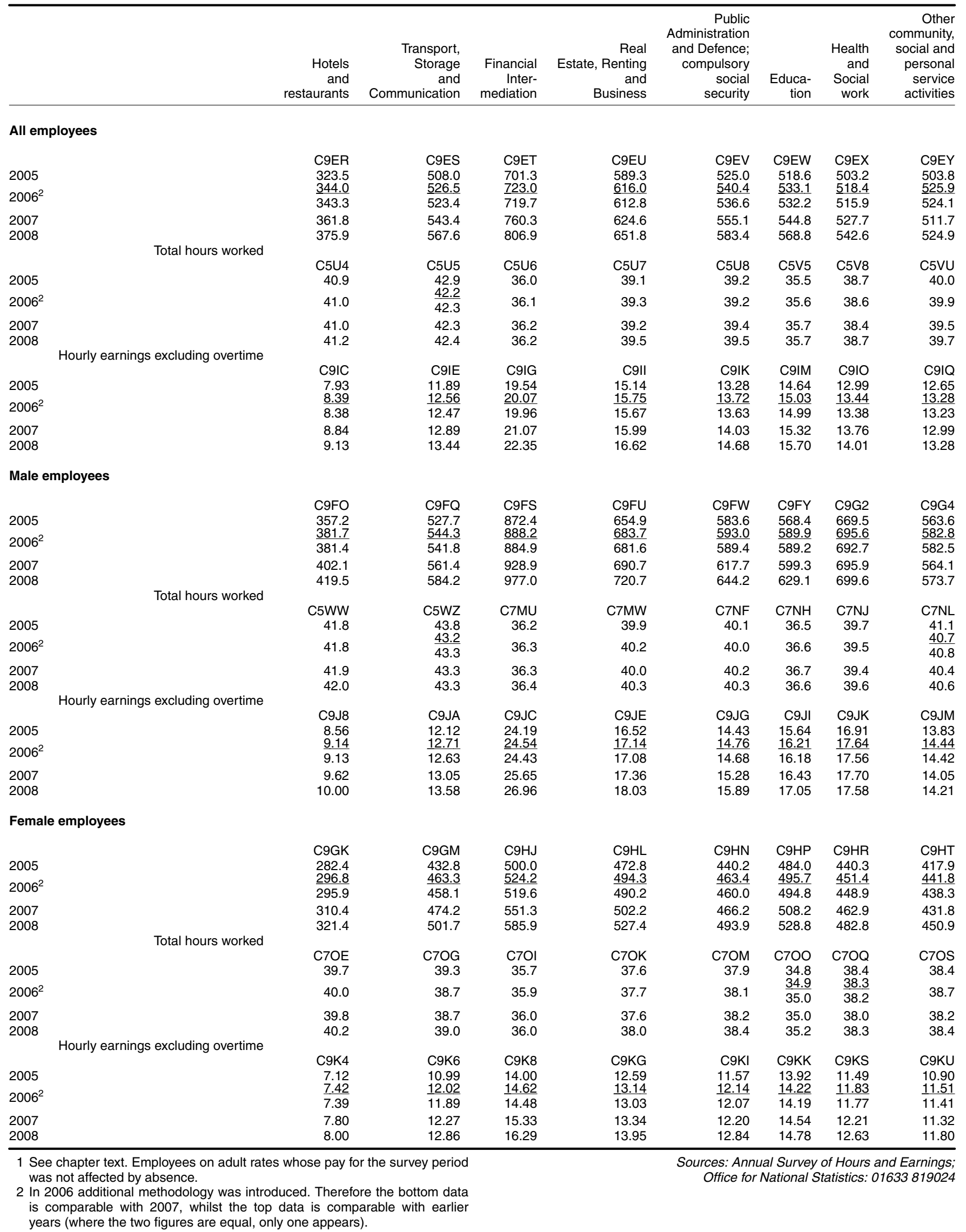


Labour Market

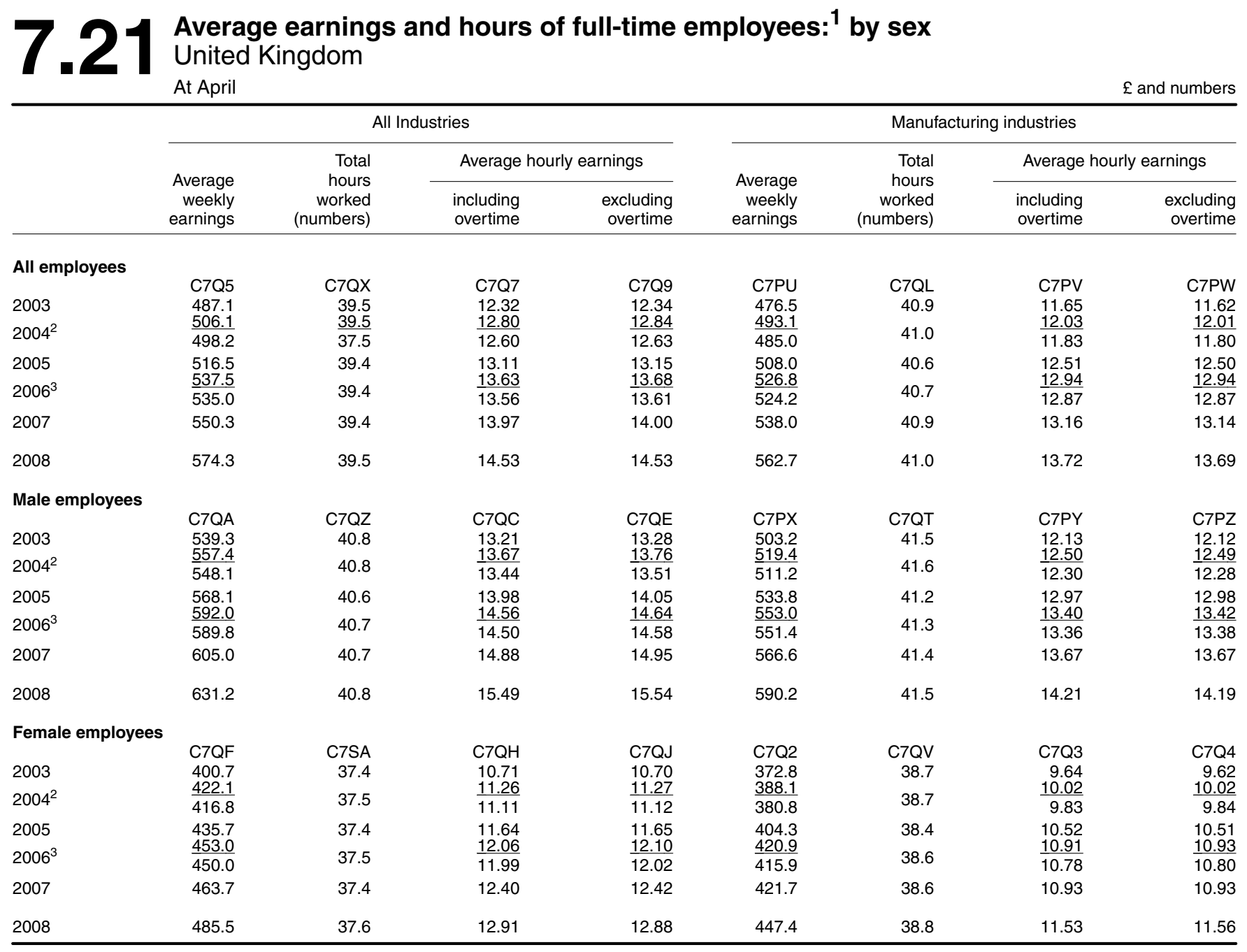

1 See chapter text. Employees on adult rates whose pay for the survey period 3 In 2006 additional methodology was introduced. Therefore the bottom data is was not affected by absence.

2 For 2004, two sets of figures are shown. The first does not include supplecomparable with 2007 , whilst the top data is comparable with earlier years mentary information and therefore is comparable with earlier years. The second includes supplementary information and so is discontinuous with (where the two figures are equal, only one appears).

Sources: Annual Survey of Hours and Earnings; Office for National Statistics: 01633819024 


\begin{tabular}{|c|c|c|c|c|c|c|c|c|c|c|c|c|c|}
\hline & \multicolumn{13}{|c|}{ Not seasonally adjusted } \\
\hline & $\begin{array}{r}\text { Annual } \\
\text { averages }\end{array}$ & $\begin{array}{l}\text { Jan- } \\
\text { uary }\end{array}$ & $\begin{array}{l}\text { Feb- } \\
\text { ruary }\end{array}$ & March & April & May & June & July & August & $\begin{array}{l}\text { Sept- } \\
\text { ember }\end{array}$ & $\begin{array}{l}\text { Oct- } \\
\text { ober }\end{array}$ & $\begin{array}{l}\text { Nov- } \\
\text { ember }\end{array}$ & $\begin{array}{l}\text { Dec- } \\
\text { ember }\end{array}$ \\
\hline \multicolumn{14}{|c|}{$\begin{array}{l}\text { Whole economy } \\
\text { (Divisions } 01-93 \text { ) }\end{array}$} \\
\hline & LNMM & & & & & & & & & & & & \\
\hline 2006 & 126.4 & 127.2 & 131.6 & 133.2 & 124.1 & 124.5 & 126.4 & 125.2 & 123.5 & 123.7 & 123.9 & 124.6 & 129.4 \\
\hline 2007 & 131.5 & 133.3 & 138.8 & 137.8 & 128.0 & 129.0 & 130.5 & 129.7 & 128.7 & 128.8 & 128.2 & 130.2 & 134.4 \\
\hline \multirow{2}{*}{\multicolumn{14}{|c|}{$\begin{array}{l}\text { Manufacturing industries } \\
\text { (Divisions } 15-37 \text { ) }\end{array}$}} \\
\hline & & & & & & & & & & & & & \\
\hline 2006 & 126.2 & 121.9 & 125.5 & 133.0 & 126.8 & 124.1 & 125.2 & 125.5 & 124.4 & 125.6 & 126.5 & 126.1 & 130.0 \\
\hline 2007 & 130.7 & 125.9 & 130.1 & 137.8 & 130.8 & 129.6 & 130.4 & 131.1 & 127.9 & 128.6 & 130.0 & 130.8 & 135.6 \\
\hline 2008 & 134.6 & 130.5 & 134.1 & 143.6 & 135.4 & 134.1 & 133.5 & 134.4 & 131.5 & 132.2 & 133.3 & 133.7 & 138.6 \\
\hline \multicolumn{14}{|c|}{$\begin{array}{l}\text { Production industries } \\
\text { (Divisions } 10-41 \text { ) }\end{array}$} \\
\hline & LNMO & & & & & & & & & & & & \\
\hline 2006 & 126.0 & 121.7 & 125.2 & 133.0 & 126.9 & 124.1 & 125.6 & 125.3 & 124.0 & 125.2 & 126.0 & 125.9 & 129.4 \\
\hline 2007 & 130.9 & 125.7 & 129.7 & 138.8 & 131.0 & 129.7 & 131.0 & 131.3 & 127.9 & 128.8 & 130.0 & 131.1 & 135.2 \\
\hline 2008 & 135.1 & 130.6 & 134.1 & 145.1 & 136.1 & 134.5 & 134.7 & 135.0 & 131.8 & 132.8 & 133.6 & 134.1 & 138.6 \\
\hline \multicolumn{14}{|c|}{$\begin{array}{l}\text { Service industries } \\
\text { (Divisions } 50-93 \text { ) }\end{array}$} \\
\hline & LNMP & & & & & & & & & & & & \\
\hline 2006 & 126.5 & 128.6 & 133.4 & 133.5 & 123.5 & 124.6 & 126.6 & 125.1 & 123.5 & 123.3 & 123.4 & 124.2 & 129.1 \\
\hline 2007 & 131.7 & 135.3 & 141.4 & 137.8 & 127.5 & 129.0 & 130.6 & 129.5 & 129.1 & 128.7 & 127.9 & 129.8 & 133.9 \\
\hline 2008 & 136.7 & 140.7 & 147.7 & 144.8 & 132.2 & 133.1 & 135.4 & 134.6 & 133.5 & 133.0 & 132.8 & 133.7 & 138.6 \\
\hline \multicolumn{14}{|c|}{$\begin{array}{l}\text { Private sector services } \\
\text { (Divisions } 50-99 \text { ) }\end{array}$} \\
\hline & JJGF & & & & & & & & & & & & \\
\hline 2006 & 125.2 & 129.2 & 135.1 & 135.3 & 121.5 & 121.6 & 125.1 & 123.0 & 121.0 & 120.8 & 121.0 & 121.7 & 127.3 \\
\hline 2007 & 130.7 & 136.7 & 144.6 & 139.8 & 125.5 & 126.1 & 129.1 & 128.1 & 127.0 & 126.8 & 125.7 & 127.1 & 131.9 \\
\hline \multirow[t]{2}{*}{2008} & 135.7 & 142.2 & 151.4 & 147.6 & 130.1 & 130.2 & 134.3 & 132.9 & 131.0 & 130.7 & 130.6 & 130.5 & 136.6 \\
\hline & \multicolumn{13}{|c|}{ Seasonally adjusted } \\
\hline
\end{tabular}

Whole economy

(Divisions 01 - 93)

$2006 \quad$ LNMQ

126.5

$\begin{array}{ll}2007 & 131.5 \\ 2008 & 136.1\end{array}$

Manufacturing industries

(Divisions 15 - 37)

$\begin{array}{lr}2006 & \text { LNMR } \\ 2007 & 126.4 \\ 2008 & 130.8 \\ & 134.8\end{array}$

Production industries

(Divisions 10 - 41)

$2006 \quad 126.0$

2007

126.0
130.7

123.8

$123.3 \quad 124.6$

$129.4-130.2$

$134.3 \quad 135.1$

$124.0 \quad 124.7$

133.2133 .2 $\begin{array}{lll}125.1 & 125.7 & 126.1\end{array}$

$\begin{array}{lll}129.4 & 130.1 & 130.8\end{array}$

$\begin{array}{lll}135.2 & 134.9 & 135.3\end{array}$
127.1

131.2

135.8
126.7

131.5
136.2

\section{9}

132.4

136.6
$127.5 \quad 128.1$

$132.9 \quad 132.5$

$137.0 \quad 137.3$
$128.2 \quad 129.0$

$133.8 \quad 133.8$ $137.5 \quad 138.1$

2008 135.1

124.7

1250

128.9

130.0

130.5
134.8

126.3

127.5

128

128.4

$128.1 \quad 128.0$

$\begin{array}{lllllll}131.2 & 131.2 & 131.1 & 131.4 & 131.7 & 132.7 & 133.3 \\ 134.4 & 134.8 & 135.0 & 135.3 & 135.6 & 135.9 & 136.3\end{array}$

$\begin{array}{llllll}134.8 & 135.0 & 135.3 & 135.6 & 135.9 & 136.3\end{array}$

Service industries

(Divisions 50 - 93)

$\begin{array}{ll}2006 & \text { LNMT } \\ 2007 & 126.7 \\ 2008 & 131.8\end{array}$

$123.1 \quad 124$

$125.7 \quad 125.7 \quad 126.4$

127

$125.9 \quad 125.4$

$133.3 \quad 133.3$

$\begin{array}{lll}124.3 & 125.8 & 125.1\end{array}$

$129.2 \quad 129.8 \quad 130.2$

131.1

131.3

127.1

127.7

128.0

Private sector services

(Divisions 50-93)

\begin{tabular}{llllllllllllll}
2006 & JJGH & & & & & & & & & \\
25.6 & 121.6 & 123.3 & 124.7 & 124.6 & 125.2 & 126.5 & 125.8 & 125.7 & 126.5 & 127.4 & 127.5 & 128.5 \\
2007 & 131.1 & 128.9 & 130.1 & 128.5 & 129.5 & 130.3 & 130.5 & 131.1 & 132.1 & 133.0 & 132.2 & 133.6 & 133.3 \\
2008 & 136.0 & 134.0 & 134.8 & 135.4 & 134.8 & 135.0 & 135.8 & 136.1 & 136.5 & 136.9 & 137.4 & 137.5 & 138.2 \\
\hline
\end{tabular}

1 See chapter text. 
Labour Market

\begin{tabular}{|c|c|c|c|c|c|c|c|c|c|}
\hline $\begin{array}{r}\text { Agriculture, } \\
\text { forestry } \\
\text { and } \\
\text { fishing }\end{array}$ & $\begin{array}{r}\text { Mining } \\
\text { and } \\
\text { quarrying }\end{array}$ & $\begin{array}{r}\text { Food } \\
\text { products, } \\
\text { beverages } \\
\text { and } \\
\text { tobacco }\end{array}$ & $\begin{array}{r}\text { Textiles, } \\
\text { leather and } \\
\text { clothing }\end{array}$ & $\begin{array}{l}\text { Chemicals } \\
\text { and man- } \\
\text { made fibres }\end{array}$ & $\begin{array}{r}\text { Basic metals } \\
\text { and metal } \\
\text { products }\end{array}$ & $\begin{array}{r}\text { Engineering } \\
\text { and allied } \\
\text { industries }\end{array}$ & $\begin{array}{r}\text { Other } \\
\text { manufacturing }\end{array}$ & $\begin{array}{r}\text { Electricity, } \\
\text { gas and } \\
\text { water supply }\end{array}$ & Construction \\
\hline
\end{tabular}

Excluding bonuses

SIC 1992

\begin{tabular}{|c|c|c|c|c|c|c|c|c|c|c|}
\hline & JVUZ & JVVA & JVVB & JVVC & JVVD & JVVE & JVVF & JVVG & JVVH & JVVI \\
\hline 2007 & 127.4 & 139.1 & 128.6 & 125.4 & 127.2 & 130.8 & 133.0 & 132.2 & 121.3 & 130.6 \\
\hline 2008 & 128.2 & 148.7 & 132.8 & 125.0 & 130.5 & 136.0 & 136.3 & 135.5 & 125.3 & 134.1 \\
\hline 2006 May & 140.2 & 128.1 & 128.4 & 123.2 & 122.0 & 126.9 & 126.3 & 125.9 & 118.3 & 127.2 \\
\hline Jun & 141.4 & 128.4 & 127.8 & 124.0 & 123.0 & 129.5 & 126.5 & 126.9 & 118.2 & 127.9 \\
\hline Jul & 137.2 & 128.7 & 128.3 & 122.8 & 121.6 & 128.4 & 126.4 & 126.5 & 118.7 & 128.2 \\
\hline Aug & 139.9 & 129.0 & 128.2 & 120.1 & 122.5 & 127.9 & 126.2 & 127.1 & 116.2 & 126.7 \\
\hline Oct & 130.3 & 131.3 & 128.2 & 122.0 & 125.1 & 129.2 & 128.8 & 127.8 & 113.0 & 129.5 \\
\hline Nov & 123.8 & 131.7 & 127.7 & 122.4 & 123.9 & 129.9 & 129.1 & 128.8 & 116.6 & 130.0 \\
\hline Dec & 130.5 & 134.7 & 130.0 & 124.4 & 125.2 & 127.9 & 128.6 & 128.6 & 114.9 & 129.3 \\
\hline 2007 Jan & 129.5 & 133.1 & 126.7 & 124.2 & 123.2 & 128.0 & 129.6 & 128.7 & 114.3 & 130.6 \\
\hline Feb & 121.7 & 132.5 & 125.2 & 125.6 & 124.9 & 129.4 & 130.6 & 129.8 & 115.1 & 129.7 \\
\hline Mar & 129.8 & 134.0 & 125.4 & 123.5 & 125.6 & 130.0 & 132.6 & 131.4 & 118.9 & 131.5 \\
\hline Apr & 133.3 & 139.6 & 127.5 & 123.0 & 125.0 & 130.3 & 133.3 & 131.7 & 118.6 & 129.8 \\
\hline May & 132.0 & 140.4 & 130.2 & 124.2 & 127.5 & 128.4 & 133.0 & 131.3 & 122.2 & 129.5 \\
\hline Jul & 124.9 & 140.3 & 128.2 & 127.6 & 127.8 & 130.8 & 134.3 & 132.6 & 124.4 & 131.6 \\
\hline Aug & 127.7 & 141.6 & 128.8 & 127.8 & 127.1 & 130.9 & 132.8 & 132.2 & 121.5 & 129.3 \\
\hline Sep & 126.6 & 140.3 & 129.1 & 126.4 & 126.6 & 131.5 & 133.1 & 133.2 & 122.5 & 130.8 \\
\hline Oct & 128.1 & 142.3 & 130.3 & 126.6 & 129.2 & 132.8 & 134.2 & 134.4 & 124.3 & 131.3 \\
\hline Nov & 126.3 & 140.8 & 130.3 & 124.4 & 129.5 & 133.5 & 134.5 & 134.7 & 127.3 & 132.0 \\
\hline Dec & 125.0 & 143.2 & 133.2 & 122.2 & 131.0 & 132.5 & 134.3 & 134.2 & 123.3 & 131.0 \\
\hline 2008 Jan & 125.4 & 145.4 & 129.3 & 125.2 & 127.9 & 135.0 & 134.3 & 133.7 & 122.3 & 131.3 \\
\hline Feb & 126.2 & 145.9 & 130.0 & 123.8 & 129.6 & 134.7 & 134.5 & 134.9 & 122.5 & 133.1 \\
\hline Mar & 129.1 & 141.0 & 133.1 & 122.6 & 129.4 & 133.0 & 136.7 & 135.4 & 121.3 & 130.8 \\
\hline Apr & 127.5 & 150.6 & 131.6 & 125.5 & 130.0 & 135.9 & 136.7 & 135.4 & 123.3 & 133.1 \\
\hline May & 127.5 & 150.7 & 133.0 & 123.2 & 130.6 & 135.6 & 136.5 & 136.0 & 124.3 & 132.8 \\
\hline Jun & 128.9 & 152.4 & 132.5 & 126.1 & 131.4 & 136.6 & 136.5 & 136.1 & 125.9 & 134.5 \\
\hline Jul & 126.2 & 150.0 & 132.4 & 126.9 & 131.4 & 137.1 & 137.2 & 135.1 & 127.2 & 135.6 \\
\hline Aug & 126.6 & 148.8 & 133.2 & 126.0 & 129.5 & 136.2 & 136.4 & 134.6 & 125.7 & 133.1 \\
\hline Sep & 127.4 & 150.4 & 133.0 & 126.6 & 130.3 & 136.5 & 135.0 & 135.6 & 127.4 & 136.1 \\
\hline Oct & 130.1 & 148.5 & 134.2 & 127.8 & 130.9 & 137.1 & 137.0 & 136.1 & 127.5 & 136.3 \\
\hline Nov & 131.3 & 149.0 & 134.0 & 127.8 & 130.4 & 138.7 & 137.9 & 136.9 & 128.0 & 136.2 \\
\hline
\end{tabular}

Percentage change on the year

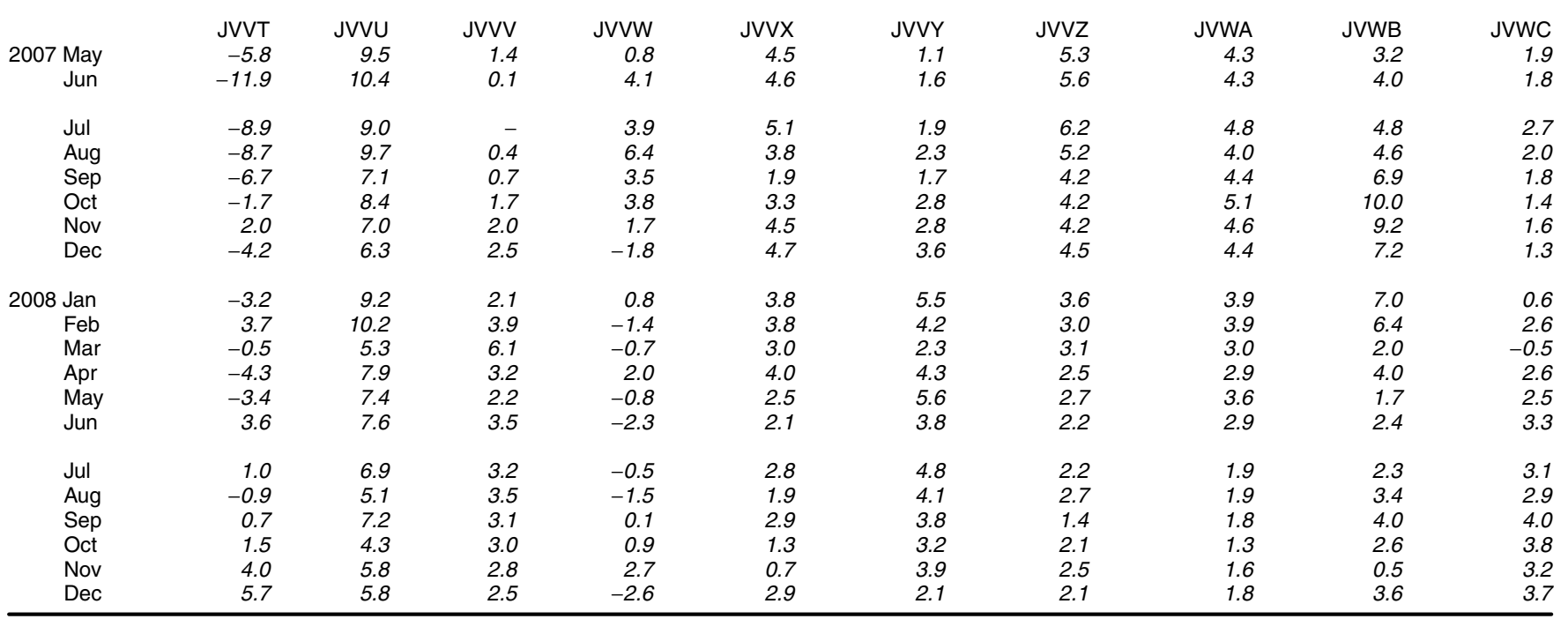


Indices $(2000=100)$

\begin{tabular}{|c|c|c|c|c|c|c|c|c|c|}
\hline $\begin{array}{r}\text { Wholesale } \\
\text { trade }\end{array}$ & $\begin{array}{l}\text { Retail trade } \\
\text { and repairs }\end{array}$ & $\begin{array}{l}\text { Hotels and } \\
\text { restaurants }\end{array}$ & $\begin{array}{r}\text { Transport, } \\
\text { storage and } \\
\text { communication }\end{array}$ & $\begin{array}{c}\text { Financial } \\
\text { interm- } \\
\text { ediation }\end{array}$ & $\begin{array}{c}\text { Real estate } \\
\text { renting and } \\
\text { business } \\
\text { activities }\end{array}$ & $\begin{array}{l}\text { Public } \\
\text { admini- } \\
\text { stration }\end{array}$ & Education & $\begin{array}{l}\text { Health and } \\
\text { social work }\end{array}$ & $\begin{array}{r}\text { Other } \\
\text { services }\end{array}$ \\
\hline
\end{tabular}

Excluding bonuses

SIC 1992

\begin{tabular}{|c|c|c|c|c|c|c|c|c|c|c|}
\hline & JVVJ & JVVK & JVVL & JVVM & JVVN & JVVO & JVVP & JVVQ & JVVR & JVVS \\
\hline 2007 & 127.2 & 122.3 & 140.3 & 130.0 & 128.6 & 133.7 & 130.7 & 134.0 & 142.7 & 126.0 \\
\hline 2008 & 131.3 & 126.9 & 144.1 & 133.6 & 133.2 & 139.5 & 135.3 & 138.4 & 149.7 & 133.3 \\
\hline 2006 May & 120.9 & 120.0 & 133.1 & 127.5 & 126.5 & 127.3 & 127.9 & 127.1 & 137.2 & 122.3 \\
\hline Jun & 122.1 & 118.5 & 132.1 & 127.9 & 125.7 & 128.0 & 128.4 & 127.6 & 138.7 & 124.6 \\
\hline Jul & 122.0 & 119.2 & 134.0 & 126.8 & 125.8 & 128.0 & 128.5 & 128.8 & 138.7 & 123.0 \\
\hline Aug & 122.1 & 120.1 & 134.1 & 126.8 & 125.6 & 128.1 & 127.2 & 131.6 & 137.7 & 122.7 \\
\hline Oct & 123.6 & 120.5 & 136.2 & 127.0 & 126.3 & 129.3 & 128.2 & 131.3 & 137.8 & 121.2 \\
\hline Nov & 124.4 & 118.7 & 136.1 & 127.4 & 125.8 & 129.4 & 128.8 & 130.9 & 139.4 & 122.3 \\
\hline Dec & 125.3 & 119.7 & 139.8 & 128.0 & 125.8 & 130.1 & 131.4 & 131.4 & 139.2 & 123.3 \\
\hline 2007 Jan & 124.8 & 122.0 & 135.9 & 127.7 & 127.1 & 130.9 & 129.2 & 130.4 & 139.8 & 124.1 \\
\hline Feb & 125.0 & 119.6 & 137.2 & 127.7 & 127.5 & 131.5 & 129.6 & 130.2 & 139.3 & 123.1 \\
\hline Mar & 126.3 & 120.9 & 138.1 & 128.2 & 126.3 & 132.5 & 129.4 & 130.5 & 139.8 & 122.7 \\
\hline Apr & 126.8 & 123.7 & 137.6 & 129.0 & 127.7 & 132.6 & 130.2 & 132.6 & 141.0 & 123.3 \\
\hline May & 127.0 & 122.7 & 140.6 & 130.0 & 128.6 & 133.9 & 130.2 & 132.5 & 142.0 & 125.8 \\
\hline Jul & 128.0 & 122.4 & 141.4 & 130.6 & 129.2 & 134.2 & 130.0 & 133.8 & 142.0 & 127.8 \\
\hline Aug & 126.9 & 124.1 & 142.4 & 131.4 & 128.6 & 134.5 & 131.6 & 136.2 & 142.8 & 128.1 \\
\hline Sep & 127.2 & 123.5 & 141.4 & 130.4 & 129.2 & 133.9 & 131.3 & 137.3 & 143.2 & 127.3 \\
\hline Oct & 128.0 & 122.8 & 141.4 & 130.3 & 129.8 & 134.9 & 130.8 & 135.9 & 144.5 & 126.4 \\
\hline Nov & 128.4 & 122.1 & 142.8 & 131.4 & 130.3 & 135.3 & 132.1 & 137.5 & 148.8 & 127.2 \\
\hline Dec & 130.1 & 122.1 & 143.5 & 132.6 & 130.2 & 136.5 & 133.4 & 138.8 & 146.9 & 128.2 \\
\hline 2008 Jan & 129.4 & 125.7 & 141.2 & 131.0 & 130.7 & 137.0 & 133.4 & 134.7 & 146.2 & 129.7 \\
\hline Feb & 129.9 & 123.9 & 141.8 & 131.5 & 131.5 & 137.9 & 134.3 & 134.7 & 145.9 & 129.0 \\
\hline Mar & 131.0 & 126.3 & 146.9 & 132.8 & 133.1 & 137.7 & 133.5 & 134.8 & 146.7 & 130.9 \\
\hline Apr & 130.9 & 127.3 & 144.4 & 133.5 & 133.5 & 138.8 & 135.3 & 136.6 & 149.1 & 130.3 \\
\hline May & 131.2 & 127.2 & 145.8 & 133.6 & 133.1 & 138.8 & 134.7 & 136.6 & 148.1 & 132.5 \\
\hline Jun & 131.1 & 127.2 & 144.0 & 134.1 & 134.5 & 139.8 & 134.9 & 136.6 & 149.3 & 134.3 \\
\hline Jul & 130.9 & 126.8 & 144.0 & 134.0 & 134.4 & 140.4 & 135.1 & 137.9 & 150.8 & 137.3 \\
\hline Aug & 130.8 & 127.8 & 146.4 & 133.5 & 133.7 & 140.3 & 134.7 & 139.5 & 153.6 & 135.8 \\
\hline Sep & 131.7 & 128.5 & 143.6 & 134.2 & 133.1 & 139.9 & 135.0 & 141.1 & 151.1 & 134.1 \\
\hline Oct & 132.5 & 128.0 & 144.5 & 135.2 & 133.1 & 140.9 & 135.0 & 140.3 & 151.2 & 134.3 \\
\hline Nov & 132.7 & 126.5 & 141.7 & 134.6 & 134.1 & 141.4 & 139.0 & 144.9 & 151.8 & 135.0 \\
\hline
\end{tabular}

Percentage change on the year

\begin{tabular}{|c|c|c|c|c|c|c|c|c|c|c|}
\hline & JVWD & JVWE & JVWF & JVYJ & JVYK & JVYL & JVYM & JVYN & JVYO & JVYP \\
\hline 2007 May & 5.0 & 2.3 & 5.6 & 2.0 & 1.7 & 5.2 & 1.8 & 4.2 & 3.5 & 2.8 \\
\hline Jun & 4.4 & 3.1 & 6.8 & 2.5 & 2.7 & 4.7 & 1.3 & 4.1 & 2.9 & 2.7 \\
\hline Jul & 5.0 & 2.7 & 5.5 & 3.0 & 2.7 & 4.8 & 1.2 & 3.9 & 2.4 & 3.9 \\
\hline Aug & 4.0 & 3.3 & 6.2 & 3.6 & 2.4 & 5.0 & 3.4 & 3.5 & 3.7 & 4.4 \\
\hline Sep & 3.9 & 2.5 & 4.9 & 1.7 & 3.5 & 4.3 & 2.2 & 3.8 & 4.0 & 4.8 \\
\hline Oct & 3.6 & 1.9 & 3.8 & 2.6 & 2.8 & 4.3 & 2.0 & 3.5 & 4.8 & 4.3 \\
\hline Nov & 3.2 & 2.8 & 4.9 & 3.1 & 3.6 & 4.6 & 2.6 & 5.0 & 6.7 & 4.1 \\
\hline Dec & 3.9 & 2.0 & 2.6 & 3.6 & 3.5 & 4.9 & 1.6 & 5.7 & 5.5 & 4.0 \\
\hline 2008 Jan & 3.7 & 3.0 & 3.9 & 2.6 & 2.8 & 4.6 & 3.2 & 3.3 & 4.6 & 4.5 \\
\hline Feb & 4.0 & 3.6 & 3.3 & 2.9 & 3.2 & 4.8 & 3.6 & 3.4 & 4.7 & 4.7 \\
\hline Mar & 3.8 & 4.5 & 6.4 & 3.5 & 5.4 & 3.9 & 3.1 & 3.4 & 5.0 & 6.7 \\
\hline Apr & 3.2 & 2.9 & 4.9 & 3.4 & 4.6 & 4.7 & 3.9 & 3.0 & 5.8 & 5.6 \\
\hline May & 3.3 & 3.6 & 3.7 & 2.7 & 3.5 & 3.7 & 3.5 & 3.1 & 4.3 & 5.3 \\
\hline Jun & 2.8 & 4.1 & 2.1 & 2.3 & 4.2 & 4.4 & 3.7 & 2.8 & 4.6 & 5.0 \\
\hline Jul & 2.2 & 3.6 & 1.9 & 2.6 & 4.1 & 4.7 & 3.9 & 3.0 & 6.2 & 7.5 \\
\hline Aug & 3.1 & 3.0 & 2.8 & 1.6 & 3.9 & 4.4 & 2.4 & 2.4 & 7.6 & 6.0 \\
\hline Nov & 3.3 & 3.7 & -0.7 & 2.5 & 2.9 & 4.6 & 5.2 & 5.4 & 2.1 & 6.1 \\
\hline Dec & 2.3 & 4.5 & 1.1 & 2.3 & 2.9 & 3.7 & 3.9 & 3.3 & 4.2 & 6.8 \\
\hline
\end{tabular}


Labour Market

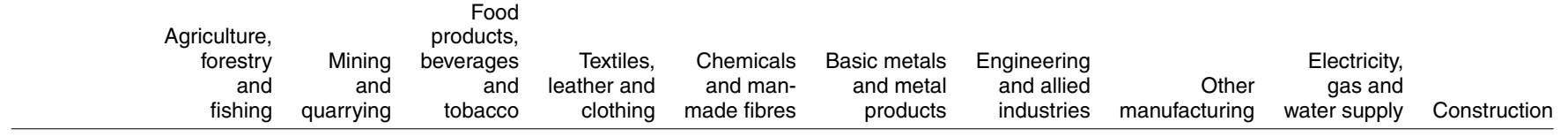

Including bonuses

SIC 1992

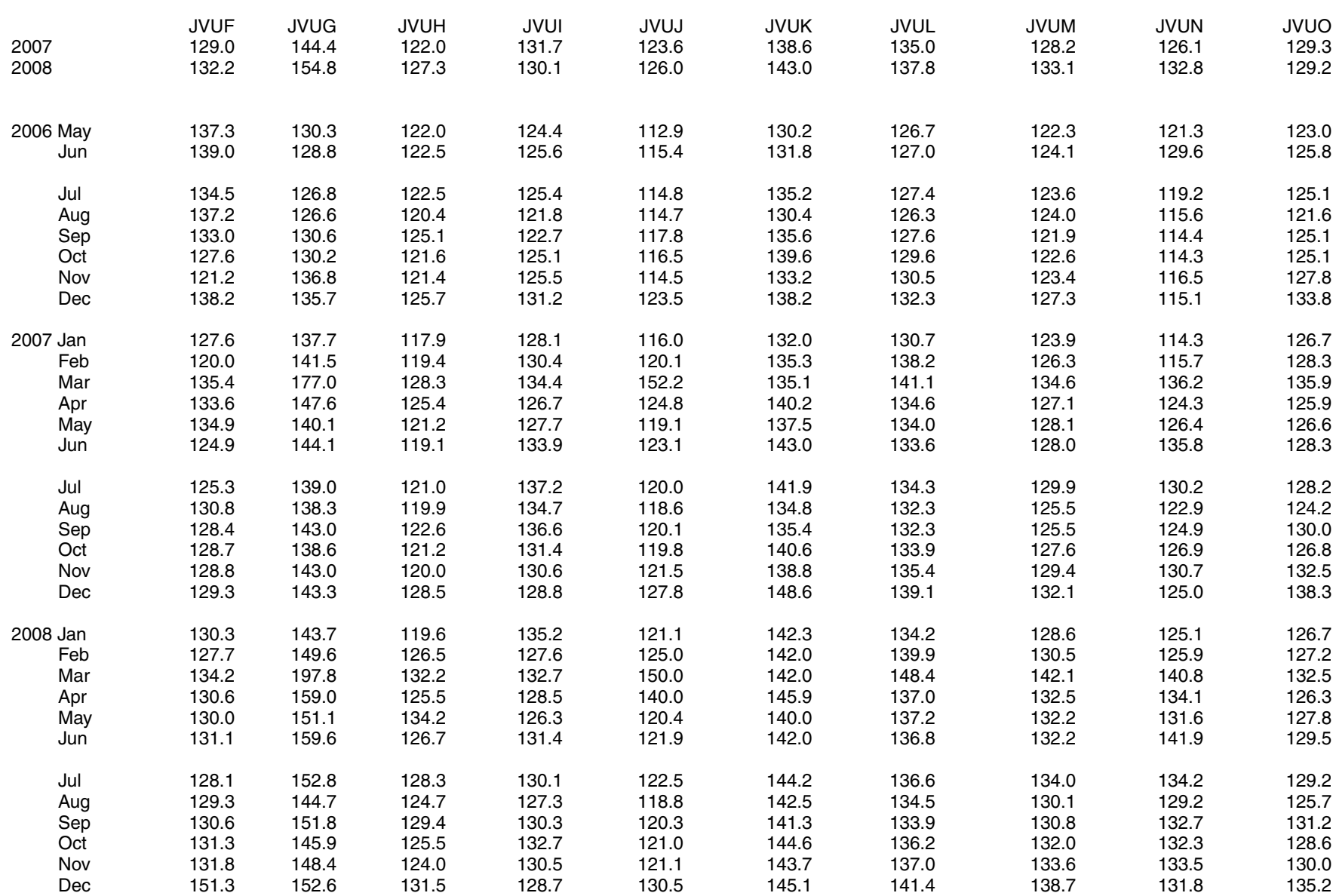

Percentage change on the year

\begin{tabular}{|c|c|c|c|c|c|c|c|c|c|c|}
\hline & JVYQ & JVYR & JVYS & JVYT & JVYU & JVYV & JVYW & JVYX & JVYY & JVYZ \\
\hline 2007 May & -1.7 & 7.5 & -0.7 & 2.6 & 5.6 & 5.6 & 5.8 & 4.7 & 4.2 & 2.9 \\
\hline Jun & -10.2 & 11.9 & -2.8 & 6.6 & 6.6 & 8.5 & 5.2 & 3.1 & 4.8 & 2.0 \\
\hline Jul & -6.8 & 9.6 & -1.2 & 9.4 & 4.5 & 5.0 & 5.5 & 5.1 & 9.2 & 2.5 \\
\hline Aug & -4.7 & 9.3 & -0.4 & 10.6 & 3.4 & 3.4 & 4.8 & 1.2 & 6.4 & 2.2 \\
\hline Sep & -3.5 & 9.5 & -2.0 & 11.3 & 2.0 & -0.1 & 3.7 & 2.9 & 9.2 & 3.9 \\
\hline Oct & 0.8 & 6.5 & -0.3 & 5.0 & 2.8 & 0.7 & 3.3 & 4.1 & 11.1 & 1.4 \\
\hline Nov & 6.2 & 4.6 & -1.2 & 4.0 & 6.1 & 4.2 & 3.8 & 4.9 & 12.2 & 3.7 \\
\hline Dec & -6.5 & 5.6 & 2.2 & -1.9 & 3.5 & 7.6 & 5.1 & 3.8 & 8.7 & 3.4 \\
\hline 2008 Jan & 2.1 & 4.3 & 1.4 & 5.5 & 4.4 & 7.8 & 2.6 & 3.7 & 9.4 & - \\
\hline Feb & 6.5 & 5.8 & 5.9 & -2.1 & 4.0 & 5.0 & 1.3 & 3.3 & 8.8 & -0.9 \\
\hline Mar & -0.8 & 11.8 & 3.0 & -1.3 & -1.5 & 5.1 & 5.2 & 5.6 & 3.3 & -2.5 \\
\hline Apr & -2.2 & 7.7 & 0.1 & 1.4 & 12.2 & 4.1 & 1.8 & 4.2 & 7.9 & 0.3 \\
\hline May & -3.7 & 7.9 & 10.7 & -1.1 & 1.1 & 1.8 & 2.4 & 3.2 & 4.1 & 1.0 \\
\hline Jun & 5.0 & 10.7 & 6.4 & -1.9 & -0.9 & -0.7 & 2.4 & 3.3 & 4.5 & 1.0 \\
\hline Jul & 2.3 & 9.9 & 6.1 & -5.2 & 2.0 & 1.6 & 1.7 & 3.2 & 3.1 & 0.8 \\
\hline Aug & -1.1 & 4.6 & 4.0 & -5.4 & 0.1 & 5.8 & 1.7 & 3.6 & 5.1 & 1.2 \\
\hline Nov & 2.4 & 3.8 & 3.3 & -0.1 & -0.3 & 3.5 & 1.2 & 3.2 & 2.1 & -1.9 \\
\hline Dec & 17.0 & 6.5 & 2.3 & -0.1 & 2.1 & -2.4 & 1.7 & 5.0 & 5.4 & -2.2 \\
\hline
\end{tabular}


Indices $(2000=100)$

\begin{tabular}{|c|c|c|c|c|c|c|c|c|c|}
\hline $\begin{array}{r}\text { Wholesale } \\
\text { trade }\end{array}$ & $\begin{array}{l}\text { Retail trade } \\
\text { and repairs }\end{array}$ & $\begin{array}{l}\text { Hotels and } \\
\text { restaurants }\end{array}$ & $\begin{array}{r}\text { Transport, } \\
\text { storage and } \\
\text { communication }\end{array}$ & $\begin{array}{c}\text { Financial } \\
\text { interm- } \\
\text { ediation }\end{array}$ & $\begin{array}{r}\text { Real estate } \\
\text { renting and } \\
\text { business } \\
\text { activities }\end{array}$ & $\begin{array}{l}\text { Public } \\
\text { admini- } \\
\text { stration }\end{array}$ & Education & $\begin{array}{l}\text { Health and } \\
\text { social work }\end{array}$ & $\begin{array}{r}\text { Other } \\
\text { services }\end{array}$ \\
\hline
\end{tabular}

Including bonuses

SIC 1992

\begin{tabular}{|c|c|c|c|c|c|c|c|c|c|c|}
\hline & JVUP & JVUQ & JVUR & JVUS & JVUT & JVUU & JVUV & JVUW & JVUX & JVUY \\
\hline 2007 & 132.6 & 123.7 & 143.8 & 131.5 & 130.2 & 129.5 & 131.2 & 133.8 & 142.6 & 129.5 \\
\hline 2008 & 137.0 & 126.8 & 147.7 & 134.4 & 136.7 & 134.4 & 135.9 & 138.3 & 149.5 & 139.2 \\
\hline 2006 May & 120.2 & 119.7 & 138.4 & 139.0 & 103.4 & 122.3 & 127.7 & 126.8 & 137.0 & 125.4 \\
\hline Jun & 123.0 & 120.8 & 134.7 & 138.2 & 113.2 & 124.7 & 129.1 & 127.3 & 138.5 & 124.9 \\
\hline Jul & 123.9 & 121.3 & 136.5 & 127.5 & 103.4 & 124.9 & 131.2 & 128.7 & 138.5 & 123.9 \\
\hline Aug & 121.3 & 119.0 & 136.9 & 124.6 & 99.3 & 122.2 & 130.1 & 131.3 & 137.4 & 123.2 \\
\hline Oct & 124.6 & 120.2 & 139.4 & 122.9 & 97.7 & 122.6 & 128.6 & 130.9 & 137.6 & 120.6 \\
\hline Nov & 126.6 & 118.0 & 140.7 & 124.4 & 100.4 & 122.7 & 129.1 & 130.7 & 139.2 & 123.5 \\
\hline Dec & 133.9 & 118.9 & 145.5 & 130.8 & 113.5 & 129.5 & 134.7 & 131.2 & 139.2 & 126.7 \\
\hline 2007 Jan & 129.1 & 120.5 & 139.3 & 125.5 & 195.0 & 125.3 & 128.9 & 130.0 & 139.8 & 126.8 \\
\hline Feb & 133.7 & 120.0 & 142.5 & 127.5 & 243.1 & 127.4 & 129.6 & 129.9 & 139.5 & 125.1 \\
\hline Mar & 140.6 & 128.4 & 146.3 & 129.7 & 176.0 & 134.4 & 129.3 & 130.3 & 140.5 & 129.2 \\
\hline Apr & 129.9 & 126.4 & 139.3 & 126.3 & 105.1 & 127.5 & 130.4 & 132.3 & 140.7 & 127.8 \\
\hline May & 128.8 & 124.1 & 145.8 & 140.9 & 108.0 & 127.2 & 130.3 & 132.1 & 141.7 & 127.8 \\
\hline Jul & 130.2 & 126.7 & 142.6 & 129.0 & 104.3 & 132.1 & 130.4 & 133.8 & 141.8 & 133.0 \\
\hline Aug & 129.0 & 122.8 & 144.6 & 130.7 & 102.7 & 129.9 & 134.4 & 135.8 & 142.5 & 131.0 \\
\hline Sep & 131.1 & 122.4 & 143.0 & 127.9 & 106.4 & 128.1 & 131.1 & 136.9 & 143.1 & 129.3 \\
\hline Oct & 130.4 & 123.6 & 143.8 & 127.7 & 99.0 & 128.1 & 130.6 & 135.9 & 144.2 & 128.4 \\
\hline Nov & 135.4 & 121.0 & 145.8 & 130.0 & 103.9 & 128.7 & 133.0 & 137.5 & 148.6 & 129.9 \\
\hline Dec & 140.4 & 122.3 & 150.4 & 137.0 & 113.1 & 135.0 & 135.6 & 138.9 & 146.8 & 133.0 \\
\hline 2008 Jan & 138.3 & 123.7 & 144.2 & 129.5 & 196.9 & 131.3 & 134.1 & 134.4 & 145.8 & 135.2 \\
\hline Feb & 143.2 & 125.3 & 149.1 & 132.5 & 250.2 & 132.1 & 134.4 & 134.7 & 145.6 & 136.3 \\
\hline Mar & 149.5 & 134.3 & 153.3 & 135.7 & 185.8 & 141.7 & 133.4 & 134.7 & 146.7 & 139.4 \\
\hline Apr & 134.6 & 128.8 & 147.0 & 132.2 & 104.6 & 133.3 & 135.5 & 136.3 & 148.8 & 138.6 \\
\hline May & 133.6 & 127.7 & 151.6 & 143.2 & 109.2 & 131.9 & 134.6 & 136.3 & 147.9 & 135.3 \\
\hline Jun & 136.0 & 128.2 & 146.2 & 144.9 & 119.3 & 134.8 & 134.7 & 136.4 & 149.1 & 139.6 \\
\hline Jul & 134.8 & 127.0 & 146.2 & 131.6 & 110.5 & 137.9 & 137.2 & 138.0 & 150.6 & 143.5 \\
\hline Aug & 132.0 & 125.1 & 148.4 & 132.3 & 109.8 & 133.5 & 137.2 & 139.1 & 153.5 & 140.5 \\
\hline Sep & 132.1 & 124.5 & 145.3 & 130.2 & 113.8 & 132.2 & 135.1 & 140.9 & 150.8 & 138.2 \\
\hline Oct & 133.3 & 126.0 & 146.1 & 131.2 & 107.6 & 133.1 & 134.5 & 140.1 & 150.9 & 140.5 \\
\hline Nov & 136.7 & 124.1 & 145.3 & 131.4 & 106.4 & 133.0 & 139.6 & 144.8 & 151.8 & 139.4 \\
\hline
\end{tabular}

Percentage change on the year

\begin{tabular}{|c|c|c|c|c|c|c|c|c|c|c|}
\hline & JVZA & JVZB & JVZC & JVZD & JVZE & JVZF & JVZG & JVZH & JVZI & JVZJ \\
\hline 2007 May & 7.2 & 3.7 & 5.4 & 1.4 & 4.5 & 4.0 & 2.0 & 4.2 & 3.4 & 1.9 \\
\hline Jun & 7.6 & 4.6 & 5.8 & 5.1 & -6.0 & 4.2 & 1.6 & 4.0 & 2.9 & 6.0 \\
\hline Jul & 5.1 & 4.5 & 4.5 & 1.2 & 0.9 & 5.7 & -0.6 & 3.9 & 2.4 & 7.3 \\
\hline Aug & 6.3 & 3.2 & 5.6 & 4.9 & 3.4 & 6.4 & 3.3 & 3.4 & 3.7 & 6.3 \\
\hline Sep & 7.6 & 2.3 & 4.0 & 2.7 & 10.0 & 4.8 & 2.0 & 3.8 & 4.1 & 6.3 \\
\hline Oct & 4.6 & 2.8 & 3.2 & 3.9 & 1.3 & 4.5 & 1.6 & 3.8 & 4.8 & 6.5 \\
\hline Nov & 7.0 & 2.5 & 3.6 & 4.6 & 3.5 & 4.9 & 3.1 & 5.2 & 6.7 & 5.1 \\
\hline Dec & 4.8 & 2.9 & 3.4 & 4.8 & -0.3 & 4.3 & 0.6 & 5.9 & 5.5 & 5.0 \\
\hline 2008 Jan & 7.1 & 2.6 & 3.5 & 3.2 & 1.0 & 4.8 & 4.0 & 3.4 & 4.4 & 6.6 \\
\hline Feb & 7.1 & 4.4 & 4.6 & 3.9 & 2.9 & 3.7 & 3.7 & 3.7 & 4.4 & 8.9 \\
\hline Mar & 6.3 & 4.6 & 4.8 & 4.6 & 5.6 & 5.4 & 3.1 & 3.3 & 4.4 & 7.9 \\
\hline Apr & 3.6 & 2.0 & 5.5 & 4.6 & -0.5 & 4.5 & 3.9 & 3.1 & 5.8 & 8.4 \\
\hline May & 3.7 & 2.9 & 3.9 & 1.7 & 1.1 & 3.7 & 3.3 & 3.2 & 4.3 & 5.9 \\
\hline Jun & 2.8 & 1.5 & 2.6 & -0.2 & 12.1 & 3.7 & 2.7 & 3.0 & 4.6 & 5.4 \\
\hline Jul & 3.5 & 0.3 & 2.5 & 2.0 & 6.0 & 4.4 & 5.2 & 3.2 & 6.2 & 7.9 \\
\hline Aug & 2.3 & 1.9 & 2.6 & 1.2 & 6.9 & 2.7 & 2.1 & 2.5 & 7.7 & 7.2 \\
\hline Oct & 2.3 & 1.9 & 1.6 & 2.7 & 8.7 & 3.9 & 2.9 & 3.1 & 4.7 & 9.4 \\
\hline Nov & 1.0 & 2.5 & -0.3 & 1.1 & 2.4 & 3.3 & 4.9 & 5.3 & 2.2 & 7.3 \\
\hline Dec & -0.3 & 3.4 & -0.3 & 0.7 & 11.6 & 2.5 & 3.5 & 3.4 & 4.2 & 8.4 \\
\hline
\end{tabular}

1 See chapter text.

Source: Office for National Statistics: 01633819024 


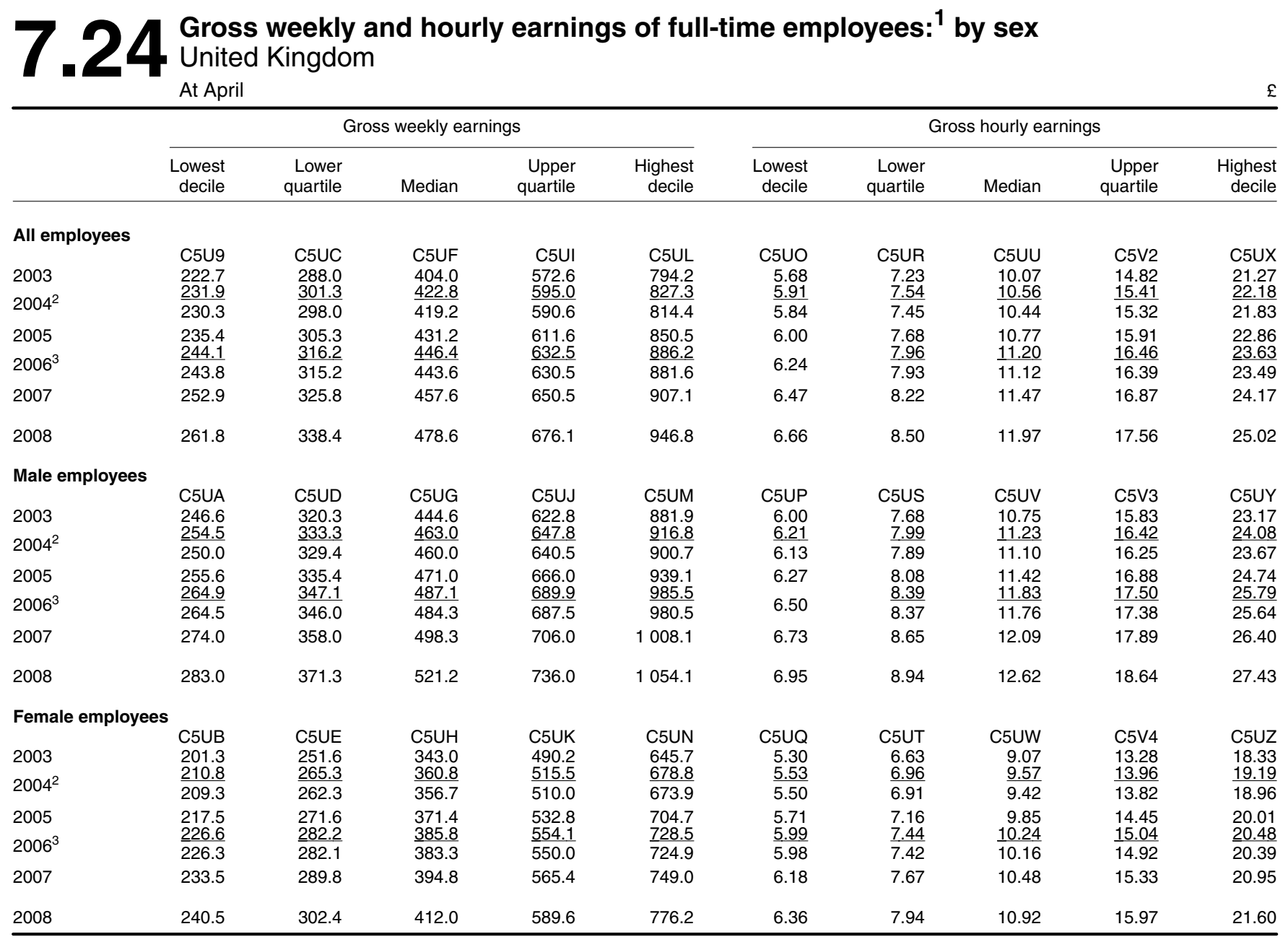

1 See chapter text. Employees on adult rates whose pay for the survey period 3 In 2006 additional methodology was introduced. Therefore the bottom data is was not affected by absence.

2 For 2004, two sets of figures are shown. The first does not include supplementary information and therefore is comparable with earlier years. The second includes supplementary information and so is discontinuous with comparable with 2007 , whilst the top data is comparable with earlier years (where the two figures are equal, only one appears).

Sources: Annual Survey of Hours and Earnings: previous years (where the two figures are equal, only one appears). 


\begin{tabular}{|c|c|c|c|c|c|}
\hline & & & & & $£$ and numbers \\
\hline & \multirow{2}{*}{$\begin{array}{r}\text { Median gross weekly pay } \\
\text { excluding overtime }\end{array}$} & \multirow{2}{*}{$\begin{array}{r}\text { Median gross weekly } \\
\text { overtime }\end{array}$} & \multicolumn{2}{|c|}{$\begin{array}{l}\text { Median weekly } \\
\text { hours (numbers) }\end{array}$} & \multirow{2}{*}{$\begin{array}{r}\text { Median gross hourly } \\
\text { earnings excluding } \\
\text { overtime }\end{array}$} \\
\hline & & & Total & Overtime & \\
\hline \multicolumn{6}{|c|}{ All employees } \\
\hline 16 to 17 & 175.9 & .. & 39.0 & .. & 4.60 \\
\hline 18 to 21 & 262.4 & 27.6 & 39.0 & 3.4 & 6.74 \\
\hline 22 to 29 & 386.0 & 41.4 & 37.5 & 3.6 & 10.11 \\
\hline 30 to 39 & 508.3 & 60.6 & 37.5 & 4.3 & 13.33 \\
\hline 40 to 49 & 507.7 & 64.9 & 37.5 & 4.7 & 13.29 \\
\hline 50 to 59 & 479.1 & 58.9 & 37.5 & 4.4 & 12.53 \\
\hline $60+$ & 408.8 & 54.6 & 38.0 & 4.8 & 10.56 \\
\hline All ages & 453.5 & 54.5 & 37.5 & 4.1 & 11.87 \\
\hline 16 to 17 & 172.5 & .. & 39.7 &.. & 4.51 \\
\hline 18 to 21 & 268.8 & 36.0 & 40.0 & 4.0 & 6.83 \\
\hline 22 to 29 & 395.7 & 49.6 & 39.1 & 4.1 & 10.11 \\
\hline 30 to 39 & 530.2 & 70.3 & 39.0 & 5.0 & 13.69 \\
\hline 40 to 49 & 559.0 & 78.7 & 39.1 & 5.5 & 14.37 \\
\hline 50 to 59 & 526.4 & 72.0 & 38.9 & 5.2 & 13.54 \\
\hline $60+$ & 429.0 & 60.7 & 39.0 & 5.0 & 10.89 \\
\hline All ages & 486.0 & 66.4 & 39.0 & 5.0 & 12.50 \\
\hline \multicolumn{6}{|c|}{ Female employees } \\
\hline 16 to 17 & 179.6 & .. & 37.5 & .. & 4.90 \\
\hline 18 to 21 & 253.9 & 18.0 & 37.5 & 2.1 & 6.62 \\
\hline 22 to 29 & 377.6 & 28.3 & 37.5 & 2.6 & 10.11 \\
\hline 30 to 39 & 472.8 & 38.1 & 37.3 & 2.9 & 12.78 \\
\hline 40 to 49 & 425.0 & 33.4 & 37.0 & 2.7 & 11.56 \\
\hline 50 to 59 & 409.0 & 28.5 & 37.0 & 2.6 & 11.08 \\
\hline $60+$ & 365.0 & 29.4 & 37.0 & 2.6 & 9.78 \\
\hline All ages & 402.5 & 30.7 & 37.1 & 2.6 & 10.91 \\
\hline
\end{tabular}

1 See chapter text. Employees on adult rates whose pay for the survey period

Sources: Annual Survey of Hours and Earnings, Office for National Statistics, was not affected by absence.

01633819024

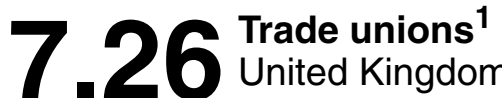 Year ending 31st March $^{2}$}

Percentages

\begin{tabular}{|c|c|c|c|c|c|c|c|c|c|}
\hline & & $\begin{array}{r}2000 \\
101\end{array}$ & $\begin{array}{r}2001 \\
102\end{array}$ & $\begin{array}{r}2002 \\
/ 03\end{array}$ & $\begin{array}{r}2003 \\
/ 04\end{array}$ & $\begin{array}{r}2004 \\
105\end{array}$ & $\begin{array}{r}2005 \\
106\end{array}$ & $\begin{array}{r}2006 \\
107\end{array}$ & $\begin{array}{r}2007 \\
108\end{array}$ \\
\hline $\begin{array}{l}\text { Number of trade unions } \\
\text { Analysis by number of members: }\end{array}$ & KCLB & 237 & 226 & 216 & 213 & 206 & 193 & 192 & 193 \\
\hline Under 100 members & KCLC & 18.60 & 22.10 & 19.00 & 20.70 & 19.90 & 17.60 & 17.70 & 17.10 \\
\hline 100 and under 500 & KCLD & 20.70 & 18.10 & 18.50 & 18.80 & 17.50 & 20.70 & 18.20 & 19.20 \\
\hline 500 and under 1,000 & KCLE & 9.30 & 9.30 & 11.60 & 10.30 & 10.70 & 9.30 & 9.90 & 10.90 \\
\hline 1,000 and under 2,500 & KCLF & 14.30 & 12.40 & 10.20 & 10.80 & 11.70 & 13.00 & 12.50 & 11.40 \\
\hline 2,500 and under 5,000 & KCLG & 9.70 & 9.30 & 11.60 & 10.80 & 10.70 & 10.90 & 11.50 & 12.40 \\
\hline 10,000 and under 15,000 & KCLI & 1.70 & 1.80 & 2.80 & 3.30 & 2.40 & 2.10 & 1.60 & 1.60 \\
\hline 15,000 and under 25,000 & KCLJ & 4.20 & 5.30 & 6.00 & 4.20 & 4.90 & 4.10 & 4.70 & 4.10 \\
\hline 25,000 and under 50,000 & KCLK & 7.60 & 6.60 & 6.50 & 7.00 & 7.30 & 7.80 & 8.90 & 8.30 \\
\hline 50,000 and under 100,000 & KCLL & 2.10 & 2.70 & 2.30 & 1.90 & 2.40 & 1.60 & 1.60 & 1.00 \\
\hline 100,000 and under 250,000 & KCLM & 2.10 & 2.20 & 2.30 & 2.80 & 2.40 & 2.60 & 2.60 & 3.10 \\
\hline 250,000 and over & KCLN & 4.60 & 4.90 & 5.10 & 4.70 & 4.90 & 4.70 & 4.70 & 4.70 \\
\hline All sizes & KCLP & 100 & 100 & 100 & 100 & 100 & 100 & 100 & 100 \\
\hline
\end{tabular}

Membership

Analysis by size of union:

KCLP

100

100

Under 100 members

100 and under 500

500 and under 1,000

1,000 and under 2,500

2,500 and under 5,000

5,000 and under 10,000

10,000 and under 15,000

15,000 and under 25,000

25,000 and under 50,000

50,000 and under 100,000

100,000 and under 250,000

250,000 and over

$\begin{array}{lrr}\text { KCLQ } & - & - \\ \text { KCLR } & 0.20 & 0.20 \\ \text { KCLS } & 0.20 & 0.20 \\ \text { KCLT } & 0.70 & 0.60 \\ \text { KCLU } & 1.10 & 1.00 \\ \text { KCLV } & 1.20 & 1.10 \\ \text { KCLW } & 0.70 & 0.60 \\ \text { KCLX } & 2.30 & 2.90 \\ \text { KCLY } & 7.80 & 6.60 \\ \text { KCLZ } & 3.80 & 4.60 \\ \text { KCMA } & 10.00 & 9.80 \\ \text { KCMB } & 72.10 & 72.40 \\ \text { KCMC } & 100 & 100\end{array}$

$\begin{array}{r}0.20 \\ 0.20 \\ 0.50 \\ 1.20 \\ 0.90 \\ 0.90 \\ 3.30 \\ 6.30 \\ 4.00 \\ 9.60 \\ 73.00 \\ \hline 100\end{array}$

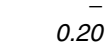

0.20

0.50
1.10

0.90

1.10

2.20

6.70

3.10

10.20

73.90

All sizes

KCMD

7897519

7779393

7750990

1 See chapter text

2 Data derived from trade union annual returns with periods which ended be-

tween October and September each year. The majority, however, ended

in December. In the case of year 2004/05, for example, the data derived from annual returns with periods which ended between October 2004 and September 2005 - approximately $73 \%$ ended in December. 